\title{
Variegation of comet 67P/Churyumov-Gerasimenko in regions showing activity
}

\author{
N. Oklay ${ }^{1}$, J.-B. Vincent ${ }^{1}$, S. Fornasier ${ }^{2}$, M. Pajola ${ }^{3}$, S. Besse ${ }^{4}$, B. J. R. Davidsson ${ }^{5}$, L. M. Lara ${ }^{6}$, S. Mottola ${ }^{7}$ \\ G. Naletto ${ }^{9,3,10}$, H. Sierks ${ }^{1}$, A. M. Barucci ${ }^{2}$, F. Scholten ${ }^{7}$, F. Preusker ${ }^{7}$, A. Pommerol ${ }^{8}$, N. Masoumzadeh ${ }^{1}$, \\ M. Lazzarin ${ }^{11}$, C. Barbieri ${ }^{11}$, P. L. Lamy ${ }^{12}$, R. Rodrigo ${ }^{13,14}$, D. Koschny ${ }^{4}$, H. Rickman ${ }^{5,15}$, M. F. A'Hearn ${ }^{16,17,1}$ \\ J.-L. Bertaux ${ }^{18}$, I. Bertini ${ }^{3}$, D. Bodewits ${ }^{16}$, G. Cremonese ${ }^{19}$, V. Da Deppo ${ }^{10}$, S. Debei ${ }^{20}$, M. De Cecco ${ }^{21}$, M. Fulle ${ }^{22}$, \\ O. Groussin ${ }^{12}$, P. J. Gutiérrez ${ }^{6}$, C. Güttler ${ }^{1}$, I. Hall ${ }^{1}$, M. Hofmann ${ }^{1}$, S. F. Hviid ${ }^{7}$, W.-H. Ip ${ }^{23}$, L. Jorda ${ }^{12}$, H. U. Keller ${ }^{24}$, \\ J. Knollenberg ${ }^{7}$, G. Kovacs ${ }^{1}$, J.-R. Kramm ${ }^{1}$, E. Kührt ${ }^{7}$, M. Küppers ${ }^{25}$, Z.-Y. Lin² ${ }^{23}$, J. J. Lopez Moreno ${ }^{6}$, F. Marzari ${ }^{11}$, \\ F. Moreno ${ }^{6}$, X. Shi ${ }^{1}$, N. Thomas ${ }^{8}$, I. Toth $^{26,12}$, and C. Tubiana ${ }^{1}$
}

(Affiliations can be found after the references)

Received 15 September 2015 / Accepted 5 December 2015

\begin{abstract}
Aims. We carried out an investigation of the surface variegation of comet 67P/Churyumov-Gerasimenko, the detection of regions showing activity, the determination of active and inactive surface regions of the comet with spectral methods, and the detection of fallback material.

Methods. We analyzed multispectral data generated with Optical, Spectroscopic, and Infrared Remote Imaging System (OSIRIS) narrow angle camera (NAC) observations via spectral techniques, reflectance ratios, and spectral slopes in order to study active regions. We applied clustering analysis to the results of the reflectance ratios, and introduced the new technique of activity thresholds to detect areas potentially enriched in volatiles.

Results. Local color inhomogeneities are detected over the investigated surface regions. Active regions, such as Hapi, the active pits of Seth and Ma'at, the clustered and isolated bright features in Imhotep, the alcoves in Seth and Ma'at, and the large alcove in Anuket, have bluer spectra than the overall surface. The spectra generated with OSIRIS NAC observations are dominated by cometary emissions of around $700 \mathrm{~nm}$ to $750 \mathrm{~nm}$ as a result of the coma between the comet's surface and the camera. One of the two isolated bright features in the Imhotep region displays an absorption band of around $700 \mathrm{~nm}$, which probably indicates the existence of hydrated silicates. An absorption band with a center between 800-900 nm is tentatively observed in some regions of the nucleus surface. This absorption band can be explained by the crystal field absorption of Fe ${ }^{2+}$, which is a common spectral feature seen in silicates.
\end{abstract}

Key words. comets: individual: 67P/Churyumov-Gerasimenko - methods: data analysis - techniques: image processing techniques: imaging spectroscopy

\section{Introduction}

Since spring 2014, the OSIRIS camera system (Keller et al. 2007) is successfully observing comet 67P/ChuryumovGerasimenko (67P), the target of ESA's spacecraft Rosetta. The camera system OSIRIS is designed to observe the comet nucleus with high spatial resolution, down to a few centimeters per pixel, to provide color information on the surface via its NAC and study gas emission in comet 67P's vicinity via its wide angle camera (WAC) with a set of 26 dedicated filters. From August 2014 until the landing of Philae on the comet's surface on November 2014, the northern hemisphere of the comet was mapped globally with spatial resolutions of $50 \mathrm{~cm} / \mathrm{px}$, down to $15 \mathrm{~cm} / \mathrm{px}$ in some areas, using various combination of filters. On February 2015, a close fly-by at $8 \mathrm{~km}$ from the surface provided us with multispectral observations at a resolution of $11 \mathrm{~cm} / \mathrm{px}$ in the Ash and Imhotep regions. Aside from determining the surface morphology in great detail using OSIRIS images (see Thomas et al. 2015b; El-Maarry et al. 2015, for morphology and region definitions), such high resolution images provided us a means to unambiguously link dust jets in the coma to a series of pits (Vincent et al. 2015).

Comet 67P globally displays a uniform gray color, except for in the regions and surface features where we see activity (i.e., the release of dust grains triggered by sublimating volatiles on the surface or sub-surface Sierks et al. 2015; Fornasier et al. 2015). The spectrum of the comet displays a red slope (Sierks et al. 2015) with some variations at smaller scales (Fornasier et al. 2015). The global spectrophotometric properties of comet 67P were investigated in detail, and three groups of terrains were identified by Fornasier et al. (2015) as low spectral slope $\left(11-14 \% / 100 \mathrm{~nm}\right.$ at phase angle of $\left.50^{\circ}\right)$, average spectral slope $\left(14-18 \% / 100 \mathrm{~nm}\right.$ at phase angle of $\left.50^{\circ}\right)$, and high spectral slope (above $18 \% / 100 \mathrm{~nm}$ at phase angle of $50^{\circ}$ ) groups. The high spectral slope group describes various parts of the comet surface and the entire Apis region. The low spectral slope group hosts the Hapi region, which was showing the most activity at the arrival to the comet on August 2014, and the Seth region, where we see jets rising from a series of active pits. The average spectral slope group includes the Ma'at region, which was mainly inactive during 2014 observations, and was covered with a thick layer of dust, but also hosts a few localized active areas.

A description of the activity rising from the Hapi region is given in Sect. B.1 with an example of a collimated jet shown in Fig. B.1. A short description of the jets detected from the pits are given in Sect. B.2 and some examples of these jets are shown in Fig. B.2. This work focuses on variegation in and around these 
Table 1. Filters of the NAC (adapted from Keller et al. 2007).

\begin{tabular}{lccc}
\hline \hline $\begin{array}{l}\text { Filter } \\
\text { name }\end{array}$ & $\begin{array}{c}\text { Wavelength } \\
(\mathrm{nm})\end{array}$ & $\begin{array}{c}\text { Bandwidth } \\
(\mathrm{nm})\end{array}$ & $\begin{array}{c}\text { Filter } \\
\text { ID }\end{array}$ \\
\hline Far-UV & 269.3 & 53.6 & F15 \\
Near-UV & 360.0 & 51.1 & F16 \\
Blue & 480.7 & 74.9 & F24 \\
Green & 535.7 & 62.4 & F23 \\
Orange & 649.2 & 84.5 & F22 \\
Hydra & 701.2 & 22.1 & F27 \\
Red & 743.7 & 64.1 & F28 \\
Ortho & 805.3 & 40.5 & F51 \\
NIR & 882.1 & 65.9 & F41 \\
$\mathrm{Fe}_{2} \mathrm{O}_{3}$ & 931.9 & 34.9 & F61 \\
IR & 989.3 & 38.2 & F71 \\
\hline
\end{tabular}

active spots: the Hapi region, pits, and alcoves in Seth and Ma' at regions; the larger Anuket alcove; the active rough terrain (with circular features) of Imhotep, including the clustered and isolated bright features (see Pommerol et al. 2015b, for the definitions of bright features) on the region. All the features investigated in this study are shown in Fig. B.3 in the investigated images.

\section{Data from OSIRIS NAC and analysis methods}

The OSIRIS NAC is equipped with filters given in Table 1, and covers the wavelength range of $250 \mathrm{~nm}-1000 \mathrm{~nm}$. We investigated the NAC images (Table 2) acquired using various filter combinations and number of images per observational sequence due to operational constrains. In this section, we describe the reduction steps carried out to generate multispectral NAC data via the images described in Table 2, and introduce the methods to analyze the comet surface.

All OSIRIS images are by default reduced with the OSIRIS standard calibration pipeline; various levels of data in various formats are generated. The calibration pipeline is described in Tubiana et al. (2015) and details of the applied calibration are given in Kovacs (in prep.). In this analysis, we only use OSIRIS NAC level 3 data that have been radiometrically calibrated and corrected for optical distortion and are in radiance units $\left(\mathrm{Wm}^{-2} \mathrm{~nm}^{-1} \mathrm{sr}^{-1}\right.$, at the central wavelengths of each filter) in planetary data system (PDS) format.

The data sets indicated in Table 2 are converted into radiance factor for further analysis via

$I / F=\frac{\pi r^{2} I_{\mathrm{m}}}{F_{\odot}}$,

where $I_{\mathrm{m}}$ is the measured intensity in radiance units, $F_{\odot} / \pi$ is solar flux at $1 \mathrm{AU}$ measured at the central wavelength for each filter, and $r$ is the heliocentric distance of the comet in astronomical units.

Surface color investigation of such images (I/F) still require two additional corrections:

1. Photometric correction to eliminate the effects due to different illumination conditions.

2. Image registration to eliminate colors created by misalignment.

\subsection{Photometric correction and image coregistration}

Since the images in different filters were taken subsequently during the observations, co-alignment is needed to produce multispectral data for further analysis. Therefore, one image per color series is selected as a reference (in bold Table 2) to be used in the image registration, and the data given in Table 2 are registered to those reference images of each series using Integrated Software for Imagers and Spectrometers (USGS ISIS3 ${ }^{1}$, Anderson et al. 2004) software, following the main steps described in Appendix A.

The photometric angles of incidence, emission, and phase are also extracted at the observing time of the same reference images for photometric correction of co-aligned images. The photometric angles are calculated with a 3D-shaped model constructed with stereophotogrammetric (SPG) methods (Preusker et al. 2015) that have been reduced from 16 million to 1 million facets for computational reasons. These now comprise a sampling distance of $8 \mathrm{~m}$ average. For surface units at the scale of $10 \mathrm{~m}$ or larger, the vertical accuracy within this reduced model is typically at the one meter scale. The SPICE kernels ${ }^{2}$ are used with SPICE toolkit for $\mathrm{C}^{3}$ (Acton 1996) for the alignment of the shape at the observing time of the reference images. Extracted photometric angles per imaging series are then imported to USGS ISIS3 software to perform photometric correction to the registered images.

Lommel-Seeliger law is a well-known description of the light scattered by low albedo bodies of the solar system in which multiple scattering of the light can be neglected (Hapke 1993). Therefore, multispectral NAC images are corrected for illumination conditions using Lommel-Seeliger disk law, included in the ISIS3 software, as follows:

$D(i, e)=\frac{2 \cos (i)}{\cos (i)+\cos (e)}$

where $D$ is the disk function with geometric angles incidence $(i)$ and emergence $(e)$. Disk corrected images are then obtained by dividing the I/F images by the disk function. In the photometrically corrected images, the regions with angles larger than $80^{\circ}$ are excluded.

The active regions of the comet surface are investigated using only photometrically corrected and registered multispectral NAC data in smaller surface regions, which are defined using regions of interest (ROI) tools of ENVI version 5.0 (Exelis Visual Information Solutions, Boulder, Colorado) software and with self-written IDL (version 8.2) procedures.

\subsection{Filter ratios and spectral slopes}

Characterization of NAC filters for the surface compositional studies were carried out (Oklay et al. 2015) with various spectral methods, which were tested with sampled laboratory spectra of the calibration materials of the instruments COmetary Secondary Ion Mass Analyzer (COSIMA), Grain Impact Analyser and Dust Accumulator (GIADA) and the most common minerals found on stardust tracks. Spectral ratio plots were found to be the best for the characterization of the comet surface and already used in the investigation of color variegation of the activity (Vincent et al. 2015). As seen in Table 2, the data containing active pits are not acquired in all NAC filters because of operational constrains. Therefore, the spectral methods proposed by Oklay et al. (2015) had to be modified and optimized according to the available filters, and additional

\footnotetext{
1 http://isis.astrogeology.usgs.gov/index.html

2 http://www.cosmos.esa.int/web/spice/

spice-for-rosetta

3 http://naif.jpl.nasa.gov/naif/toolkit.html
} 
Table 2. Color images from NAC used in this study.

\begin{tabular}{|c|c|c|c|c|c|c|c|}
\hline $\begin{array}{l}\text { Image } \\
\text { ID }\end{array}$ & Regions & $\begin{array}{c}\text { Acquisition } \\
\text { date }\end{array}$ & UT & Filters & $\begin{array}{c}r \\
(\mathrm{AU})\end{array}$ & $\begin{array}{l}\text { Resolution } \\
(\mathrm{m} / \mathrm{px})\end{array}$ & $\begin{array}{c}\text { Phase } \\
\text { angle }\left({ }^{\circ}\right)\end{array}$ \\
\hline$\# 1$ & $\begin{array}{l}\text { Seth, Hapi, } \\
\text { Anuket }\end{array}$ & $2014-08-22$ & $01: 41: 55$ & F16, F24, F23, F22, F27, F28, F51, F41, F71 & 3.51 & 1.21 & 34.84 \\
\hline$\# 2$ & Ma'at & 2014-09-12 & $01: 33: 44$ & F16, F24, F23, F22, F41 & 3.38 & 0.55 & 70.45 \\
\hline \#3 & Seth & 2014-09-20 & $14: 34: 34$ & F16, F24, F23, F22, F41 & 3.33 & 0.52 & 62.76 \\
\hline \#4 & Imhotep & 2014-09-05 & $06: 36: 14$ & F16, F23, F22, F27, F28, F41, F71 & 3.43 & 0.76 & 57.76 \\
\hline
\end{tabular}

Notes. Acquisition time is given for the reference image. Reference images are indicated in bold. Image IDs are used as a reference in all images shown in this work.

definitions had to be introduced using the observed spectral features on the comet surface. There are only four filters (F16, F23, F22 and F41) in common to be used for the comparison of all observations given in Table 2.

Spectral slopes are used in the investigation of the global colors of comet 67P (Fornasier et al. 2015), the investigation of the selected landing site J (La Forgia et al. 2015), the investigation of the landing site candidate A (Pajola et al. 2015), and the investigation of the landing site Abydos (Lucchetti et al. 2016). Spectral slopes are not only well suited for the determination of the large scale color units of the comet surface, but are also useful for the comparison of our results with previous studies. Therefore, even though this method is insensitive to the information hidden in the spectrum and is using only information given in two wavelength positions on the spectra, it is one of the methods used to ensure there is a consistent investigation of the regions hosting active features.

Spectral slopes used in this study are defined as

Spectral slope $[\% / 100 \mathrm{~nm}]=\frac{\left(R_{\mathrm{NIR}}-R_{\mathrm{Green}}\right) \times 10000}{\left(\lambda_{\mathrm{NIR}}-\lambda_{\mathrm{Green}}\right) x\left(R_{\mathrm{Green}}\right)}$,

where $R$ are the radiance factors in given filters near-IR (NIR) and Green with their central wavelengths $\lambda$.

\subsection{Decorrelation stretch}

Multispectral data that are spectrally featureless are highly correlated, therefore, the red giant branch (RGB) images generated with such data sets are usually monochromatic. Stretching RGB channels of images can emphasize the differences on the image but often not enough to detect small variations. There are various techniques that decrease the correlation (exaggerate the least correlated parts of the data set selectively) of such data sets to display data in more colors. One of these methods is decorrelation stretching, which is based on the principal component (PC) transformation of the data; this method contrast-stretches the transformed data and retransforms the PC images to the original coordinates. Details of this method is discussed in Gillespie et al. (1986). In this analysis, decorrelation stretch is applied to the images taken in various filters, using the ENVI decorrelation stretch transformation tool, and is used in the RGB channels of the displayed images. This is a common approach for the detection of various surface units of the planetary surfaces such as Earth (see Gillespie et al. 1986, for examples) and Mars (see Bandfield et al. 2004, for description). The same technique was also previously applied to the asteroid Lutetia images taken with the OSIRIS NAC (El Maarry et al. 2012).

\subsection{Laboratory data of icy mixtures}

Laboratory experiments of icy mixtures studied by Pommerol et al. (2015a) were used in the investigation of bright features observed on the surface of comet 67P (Pommerol et al. 2015b). In this study, we adopted the reflectance spectra of the ROI1 in the "comet 1" run described in Pommerol et al. (2015a) in the NAC observing range, and sampled them using NAC filter transmissions as described in Oklay et al. (2015). The spectral ratio technique proposed in Sect. 3 is then applied to the sampled laboratory spectra. This plot is then used for the investigation of the active comet surface, and, in particular the bright features of Imhotep region.

\subsection{Cluster analysis}

Classification of the surface regions determined by various methods requires reliable grouping of them to detect similar/dissimilar subregions. Therefore, IDL multivariate analysis procedures are used for the hierarchical clustering analysis of the spectral ratio plots generated with the mean spectra of the selected subregions. We use Euclidean distance in measuring the distance between data points and the nearest neighbors are used for linking the clusters. The distance here is the measure of the similarity (in smaller values) and differences (in large values). Results of cluster analysis are shown using dendrogram representation.

\section{Results}

In the analysis of the images given in Table 2, we use multispectral data, which are reduced and generated by the steps described in Sect. 2. The regions showing activity (see Figs. B.1 and B.2 for the location of the active regions) are investigated in ROIs, which are defined using both spectral slopes and decorrelation stretched RGB images. Resistant mean spectra (hereafter mean reflectance spectra) excluding data points deviating by more than $\pm 3 \sigma$ of the median per investigated ROI are then calculated for further analysis. Mean reflectance spectra of each ROI are displayed and discussed using relative reflectance profiles. Spectral ratio technique is applied to the mean reflectance spectra of each ROI and the spectral ratio plots are then used for the detection of active, inactive, and mixed surface regions. Cluster analysis is applied to the values in spectral ratio plots to determine different regions on the comet surface we analyzed.

\subsection{General view on active regions}

General characterizations of the investigated regions are carried out using the spectral slopes of the regions (Fig. 1) and 

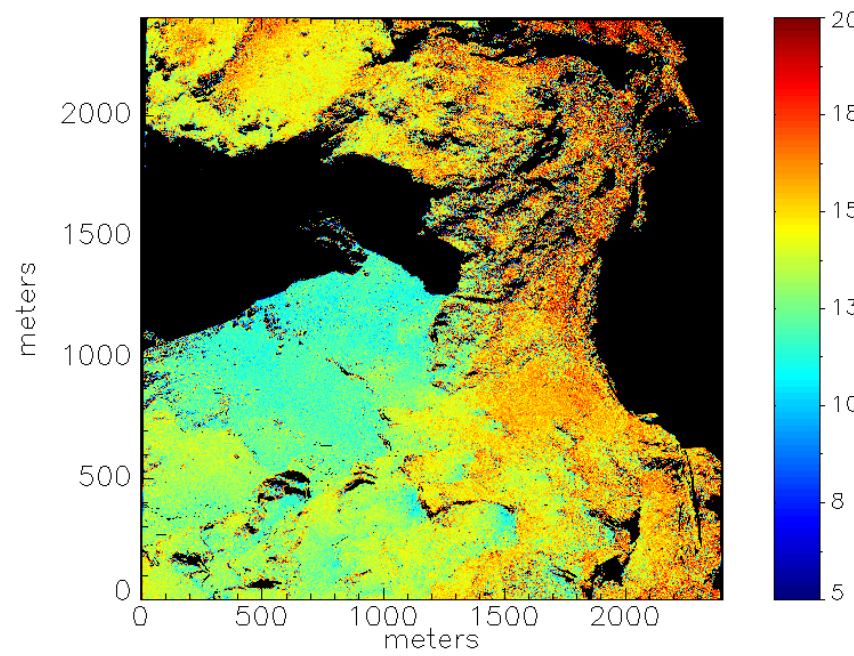

(a) Seth pits and alcoves, Hapi, large alcove of Anuket (image \#1, Fig. 3b).

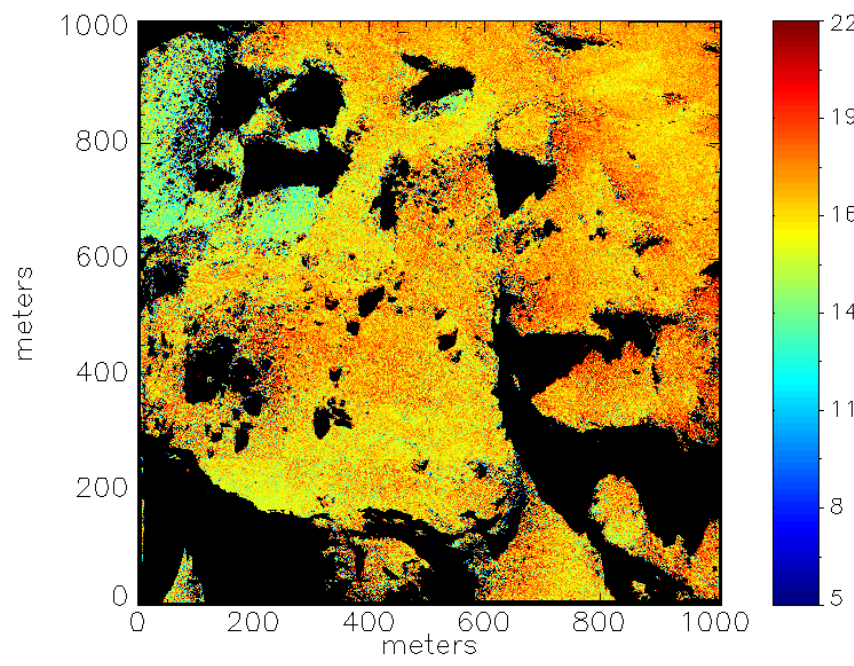

(c) Ma'at pits and alcoves (image \#2, Fig. 7b).

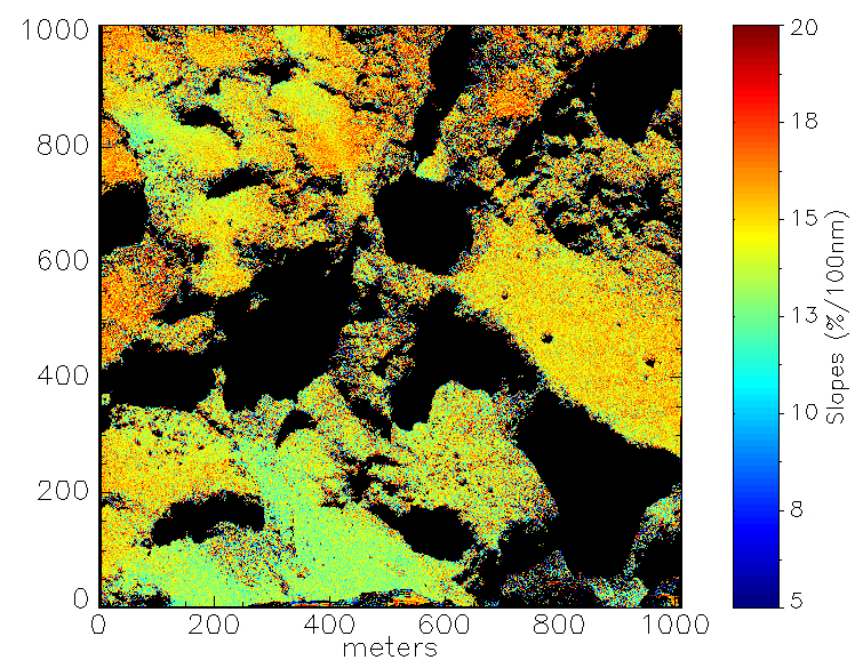

(b) Seth pits and alcoves (image \#3, Fig. 5b).

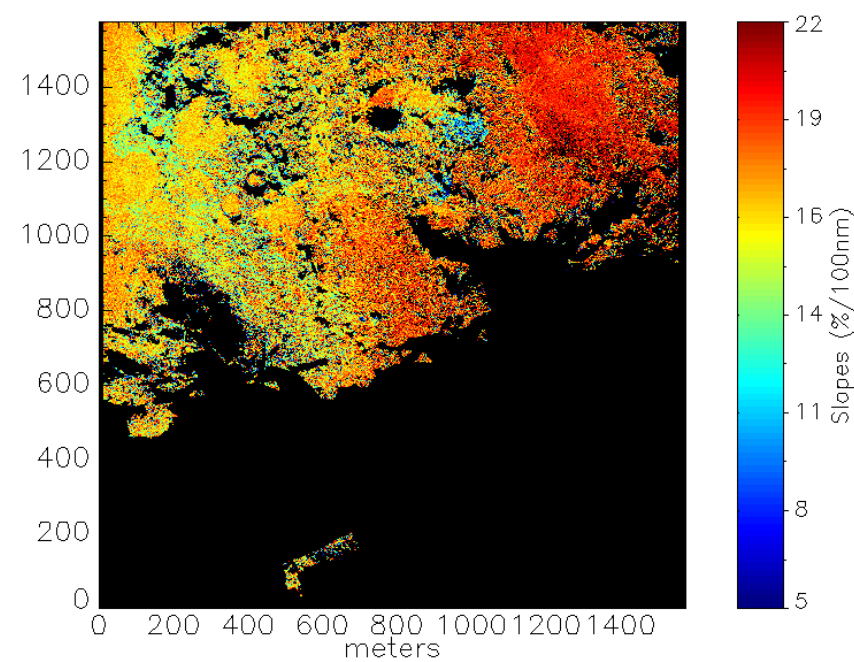

(d) Circular features, isolated, and clustered bright features of Imhotep (image \#4, Fig. 9b).

Fig. 1. Slopes of the investigated regions. The image details can be found with corresponding image ID in Table 2.

decorrelation stretched images (Fig. 2). Examples of regions where the activity was detected visually in the investigated images are given in Figs. B.1 and B.2 together with their locations on the images in Fig. B.3.

Figure 1 shows that in the regions where activity was detected visually, i.e., Hapi, Seth, and Ma'at pits have lower $(8-13 \% / 100 \mathrm{~nm})$ spectral slopes than the rest of the comet surface $(13-22 \% / 100 \mathrm{~nm})$. In addition to those places, Seth alcoves, the wall of the large Anuket alcove, around the circular features, both clustered and isolated bright features (see Thomas et al. 2015b; Auger et al. 2015; Pommerol et al. 2015b, for definitions) show similar lower spectral slopes than the rest of the surface, even though there was no visual detection of activity features rising from them at the time of the observations used in this study ${ }^{4}$. This may be because the observing geometry was not suited for their detections during the observations. In the regions

\footnotetext{
4 See Sect. 4 for the activity detected visually in the regions about half a year after the observations used in this study and for the activity detected in the regions via inversion methods.
}

we investigated, the Hapi region displays the lowest spectral slopes $8-11 \% / 100 \mathrm{~nm}$ (see also Fornasier et al. 2015) together with the isolated bright features (IBFs) and the clustered bright features in the Imhotep region. The locations of the bright features on the Imhotep image (image \#4) are shown in Fig. B.4. According to the spectral slope values, the IBFs of Imhotep seem to be more similar to the Hapi region than the active pits of Seth and Ma'at regions. Active pits, alcoves, and the large alcove of Anuket have slope values of typically 10-13\%/100 nm. The Ma'at region, which is located on the smaller lobe (head) of the comet, displays higher spectral slope values than the Seth region, which is located on the larger (body) lobe of the comet. In the investigated regions, the highest slope values are detected in the Imhotep region (see Fig. 1d). Here it should be mentioned that the comparison of spectral slopes is performed under the assumption of no spectral reddening between the phase angles of the images we investigated, although the spectral slopes show reddening by phase as presented in Fornasier et al. (2015). Unfortunately, the previous work does not cover all the phase 


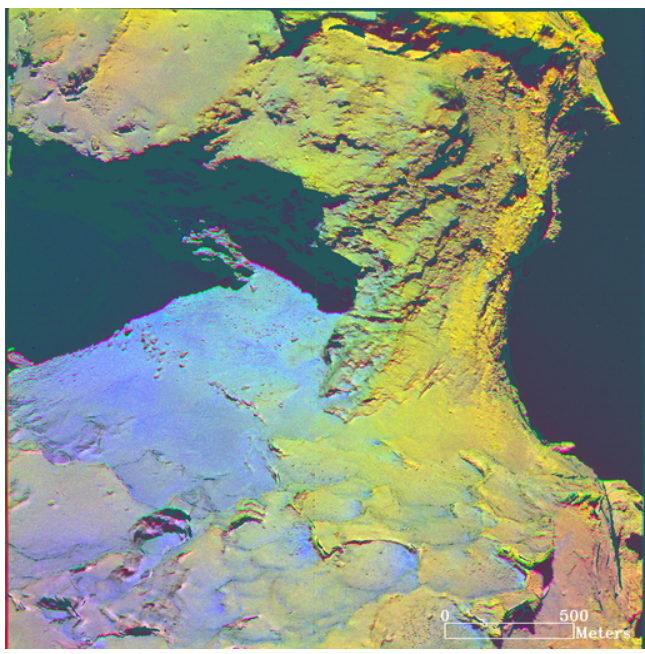

(a) Seth, Hapi, Anuket (image \#1) in R: NIR, G: Orange, B:Blue.

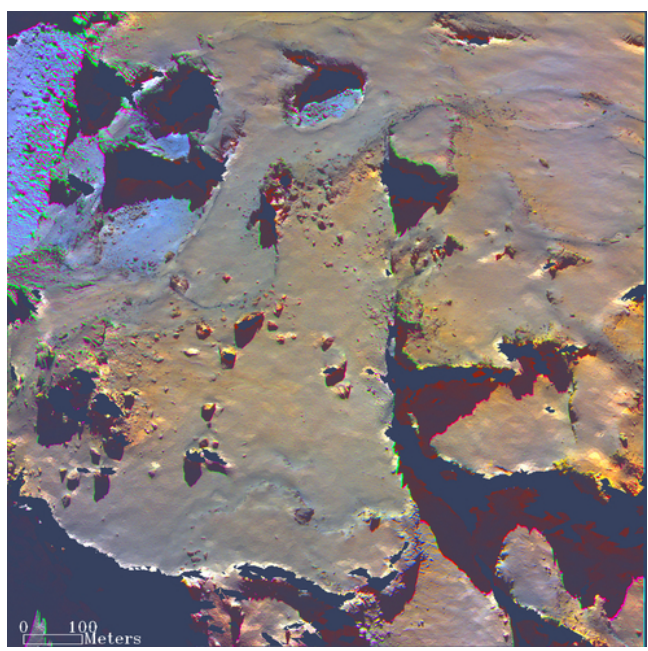

(c) Ma'at (image \#2) in R: NIR, G: Orange, B:Blue.

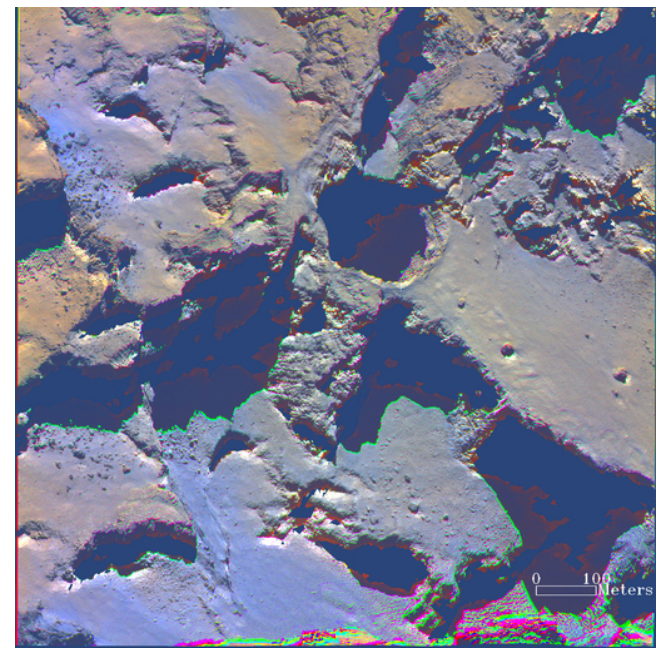

(b) Seth (image \#3) in R: NIR, G: Orange, B:Blue.

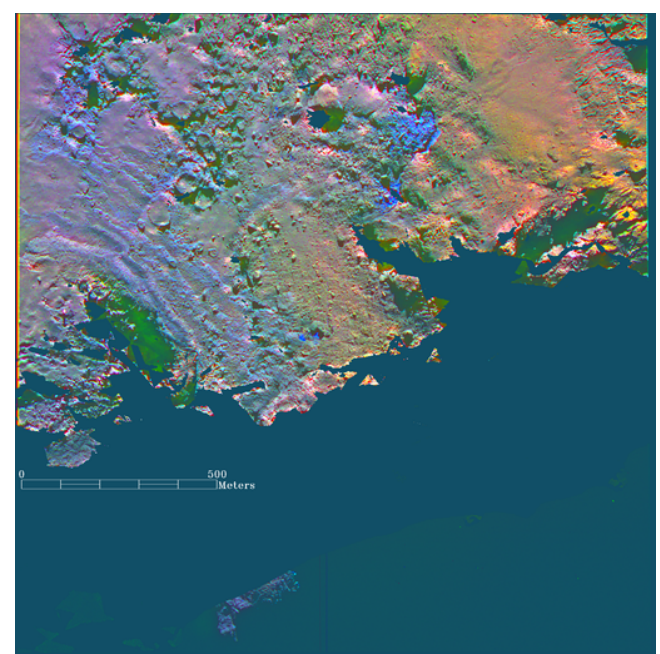

(d) Imhotep (image \#4) in R: IR, G: Hydra, B:Green.

Fig. 2. Decorrelation stretched images of active regions with RGB channels presented in subcaptions of each image. See caption of Fig. 1 for regions and features. Image details can be found with corresponding image ID in Table 2.

angles of the images we investigated, but the spectral slope variation between $35-54^{\circ}$ (Fig. 3 of Fornasier et al. 2015) is small so that we can make this comparison. However, if we follow the linear trend of the phase reddening, for the image taken in $70.45^{\circ}$ phase angle (image \#4), the spectral slopes would vary from $15 \% / 100 \mathrm{~nm}$ to $18 \% / 100 \mathrm{~nm}$ in the observations we used.

Decorrelation stretched images displayed in Fig. 2 allow much better visualization and detection of the surface variegation than the spectral slopes. Smaller scale variations around Seth pits (Fig. 2a) and in the Imhotep region (Fig. 2d), which were not caught by the spectral slopes, are instead clearly seen when this technique is used. In such RGB images, the regions where we visually detect activity, i.e., Hapi, pits of Seth, and Ma'at appears blue and purple, while the nonactive surface is yellow and orange. Similar to the active features, Seth alcoves, the large wall of the Anuket alcove, IBFs, and clustered bright features of the Imhotep region are also seen in blue and purple in the decorrelation stretched images (Fig. 2). Other than the IBFs, activity detection on these features occurred either visually (see Fig. 2c) or via inversion methods (Vincent et al. 2016; Knollenberg et al. 2015). The part of Imhotep covered by round features are purple, while those round features are surrounded by green.

Using spectral slopes and decorrelation stretched images, three surface regions are detected:

1. Active surface: regions with low spectral slopes $(8-13 \% / 100 \mathrm{~nm})$. They correspond to the regions where we detect activity: active pits, Hapi, alcoves, and bright features in Imhotep. These are also the regions blue in the decorrelation stretched images.

2. Inactive surface: regions with high spectral slopes (above $15 \% / 100 \mathrm{~nm}$ ). They correspond to the inactive surface, which are orange in the decorrelation stretched images.

3. Mixed surface: regions with intermediate spectral slopes $(13-15 \% / 100 \mathrm{~nm})$. They correspond to the regions characterized either by surfaces partially covered by material ejected from active spots or inactive areas peppered with small bright features, which are currently interpreted as minor ice deposits (Pommerol et al. 2015b). These are the regions in light orange and green in the decorrelation stretched images.

The spectral slopes of active surface correspond to the low spectral slope group (11-14\%/100 nm) defined by 
Fornasier et al. (2015) at a phase angle of about $50^{\circ}$ and at a lower spatial resolution $(>2 \mathrm{~m} / \mathrm{px})$. The regions Hapi, Seth, and portions of Ma'at, which we found to be active, belong this group, while inactive surfaces correspond to the groups with spectral slopes beyond 14\%/100 $\mathrm{nm}$ according to Fornasier et al. (2015). The mixed surface is included in both low and average surface groups of Fornasier et al. (2015), and the high spectral slope group represents the inactive surface.

\subsection{Seth and Hapi regions}

Hapi is the region in between the head and body of the comet. This is also the region that was displaying the most activity (see Fig. B.1 for an example) at the arrival to the comet (August 2014). The Seth region hosts several pits and alcoves in which emitting jets were detected (Vincent et al. 2015, 2016). An example of a jet rising from one of the Seth pits is displayed in Fig. 2a. The images we investigate (images \#1 and \#3 in Table 2) contain five active pits and many alcoves (Fig. B.3, panels a and $b$ ).

Figure 3 a displays the simulated WAC image as a context for image \#1 (details in Table 2) with NAC field of view (FOV) marked with a white square to present the location of the images on the comet surface. Figure $3 \mathrm{~b}$ presents the regions Hapi, Seth, and Anuket in the images taken using IR, hydra, and blue filters, which are coded in RGB channels of the image, respectively. The regions where we see activity (Hapi, pits, and alcoves in Seth and the large alcove in Anuket) are brighter than the rest of the visible surface, as also seen by Fornasier et al. (2015). These regions display blue-gray in this RGB scheme, while the rest of the surface is seen in gray-brown. The terrace next to Seth pits and the region around alcoves have a lighter color than the rest of the surface (see Fig. B.3, panels a and b, for the location of features). This must be because of the redistribution of the material, which was lifted up as a result of the activity and landed back on the surface (Thomas et al. 2015a). These regions are displaying the mixed material regions. Selected ROIs are enumerated and shown in Fig. 3c with their sizes given in Table C.1.

Mean reflectance spectra of each ROI displayed in Fig. 3c are calculated and presented in Fig. 4a. All regions have a similar red spectral behavior, which is the reflectance is increasing with the wavelength. Active regions are brighter, while the inactive surface and the large alcove in Anuket region are darker. All the spectra show an increase of the reflectance in the hydra and red filters (700-750 nm), that, as shown in Fornasier et al. (2015, Fig. 12 and discussion therein), is attributed to cometary emissions of the coma located between the spacecraft and the nucleus, and potentially attributed to $\mathrm{H}_{2} \mathrm{O}^{+}$and $\mathrm{NH}_{2}$. For a direct comparison, the mean reflectance values of the selected ROIs are normalized at $480.7 \mathrm{~nm}$ (blue filter) and presented in Fig. 4b. The ROIs \#1 (Seth pits), \#2 (part of Hapi close to Seth pits) and \#5 (part of Hapi toward Anuket region), where the activity was detected, are clustering with their spectra bluer than the rest of the ROIs.

Spectral ratios between various filters are investigated with the mean reflectance spectra of each ROI. The ratios between orange, hydra and hydra, NIR determined to be those achieving the best separation on the ROIs as seen in Fig. 4c. The regions where we see activity (ROIs \#1, \#2, \#5) are well separated from the rest of the ROIs with their high spectral ratio values. Inactive surface (ROIs \#6, \#7) have low spectral ratio values. ROIs \#3 and \#4 have spectral ratio values in between the active and inactive surface. The spectral ratio of the large Anuket alcove (ROI \#8) is well separated from the rest of the ROIs. This
ROI is discussed in more detail during the clustering analysis described in the following.

The clustering analysis, applied to the data shown in the spectral ratio plot (Fig. 4c), is presented using dendrogram in Fig. 4d. This analysis detects four main groups of ROIs:

1. ROI \#8: Large Anuket alcove wall (mixed region);

2. ROIs \#1, \#2, \#5: Seth pits and Hapi (active regions);

3. ROIs \#3, \#4: Seth terrace and region hosting Seth alcoves (mixed regions); and

4. ROIs \#6, \#7: Inactive surface.

The clustering analysis indicates two types of active regions: 1 . The bottom of the Seth (ROI \#1) pits and the part of the Hapi region closer to the pits (ROI \#2), which have similar spectral features; 2 . The rest of the Hapi region (ROI \#5) with its higher spectral ratios. We discuss these two types of active regions more during the analysis of the Imhotep region in Sect. 3.4. The clustering analysis also emphasizes two types of mixed regions: 1 . The vicinity of Seth pits (ROIs \#3 (Seth terrace), \#4 (the region including Seth alcoves)), which are covered with deposited material (Thomas et al. 2015a), and hosting alcoves displaying similar bright blue material as in the Seth pits and Hapi. 2. The bright large alcove of Anuket (ROI \#8) region, where bright blue active material is exposed in some parts of the inactive surface material. Activity rising from this alcove was detected via inversion of the dust jets presented in Vincent et al. (2016). The large area of the ROI \#8 might be preventing detection of the activity with the methods we use. ROIs \#6, \#7 are grouped together and represent the regions containing mostly inactive surface regions.

Seth pits and their close vicinity are investigated in a higher resolution $(0.52 \mathrm{~m} / \mathrm{px})$ multicolor image set. The simulated view representing these observations of the Seth region (image \#3 in Table 2) is presented in Fig. 5a as a context. The corresponding observation is shown in Fig. 5b, where images taken with NIR, orange and blue filters are assigned to the RGB channels of each image, respectively. The selected ROIs are presented in Fig. 5c with their size information given in Table C.2. These high resolution observations are missing the spectral information between orange and NIR filters $(649.2 \mathrm{~nm}$ and $882.1 \mathrm{~nm}$, respectively). Relative reflectance of the mean spectra of the ROIs, normalized at $480.7 \mathrm{~nm}$ (blue filter), shown in Fig. 6b are almost identical; they are featureless and have red slopes (increasing reflectance in wavelength). In this set, we have images taken only in five filters and this limits the spectral ratio analysis. The best separation of ROIs is achieved with of blue/green and orange/NIR. It is difficult to detect the three surface units, i.e., active, inactive and mixed, of defined ROIs using these ratios (see Fig. 6c), but the detection of the active regions can be reliably carried out. Active pit (ROI\#1) is well separated from the rest of the ROIs with its higher ratios. The ROIs \#2 and \#4 are clustered together, and, according to their spectral ratio values, they must show activity features as well. Even though there was no visual detection of activity in these regions, dust jet inversions by Vincent et al. (2016) detects activity in them. ROIs \#3 and \#11 seem to be mixed surface, while it is not possible to classify the rest of the ROIs since they are not grouped in this spectral ratio plot. The clustering analysis applied to the data shown in the spectral ratio plot (Fig. 6c) is presented using dendrogram in Fig. 6d. This analysis indicates four groups of ROIs:

1. ROI \#1: Seth pit (active region);

2. ROIs \#2, \#4: Seth alcoves (active regions);

3. ROIs \#3, \#5, \#11: Mixed regions; and

4. ROIs \#6-\#10: Inactive surface. 
N. Oklay et al.: Variegation of comet 67P's active regions

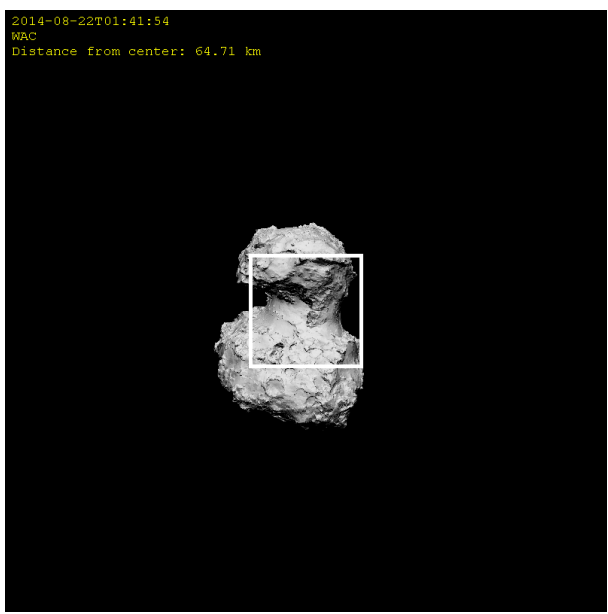

(a) WAC view of the image \#1 with the NAC field of view indicated with white square.

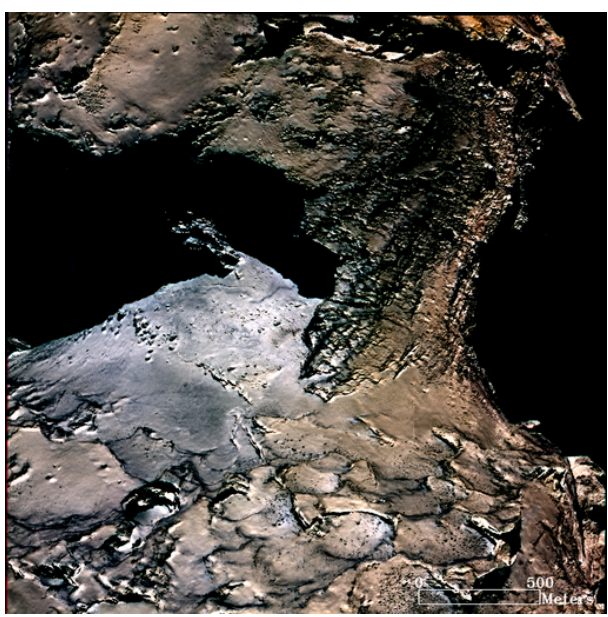

(b) Seth, Hapi, Anuket regions in image \#1. R: IR, G: Hydra, B: Blue.

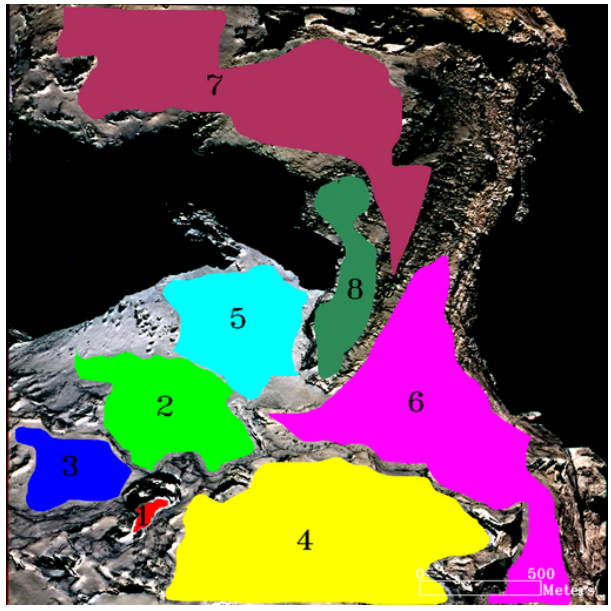

(c) Same RGB images as Fig. 3b, with region of interests overlaid.

Fig. 3. View of the image \#1 (top panel). RGB colors of Seth pits with their surrounding and Hapi region (middle panel) together with defined region of interests (bottom panel). Image details can be found with corresponding image ID in Table 2.

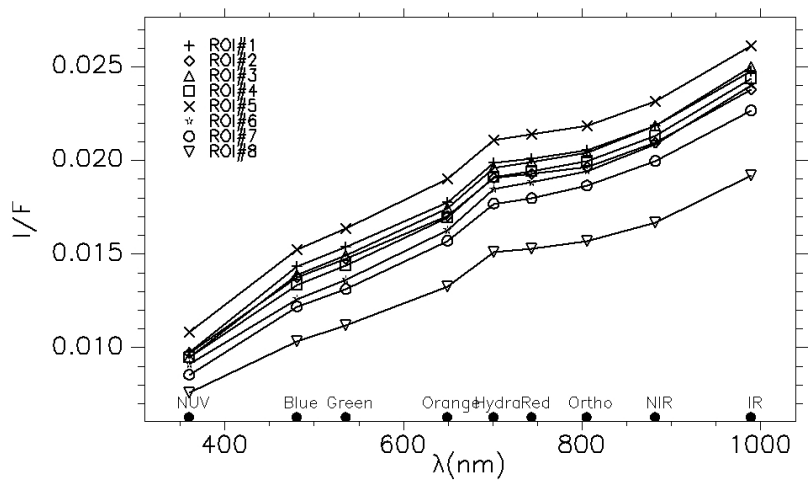

(a) Mean reflectance spectra of ROIs. Typical standard deviation of the data points from the resistant mean is $7.8 \times 10^{-6}$.

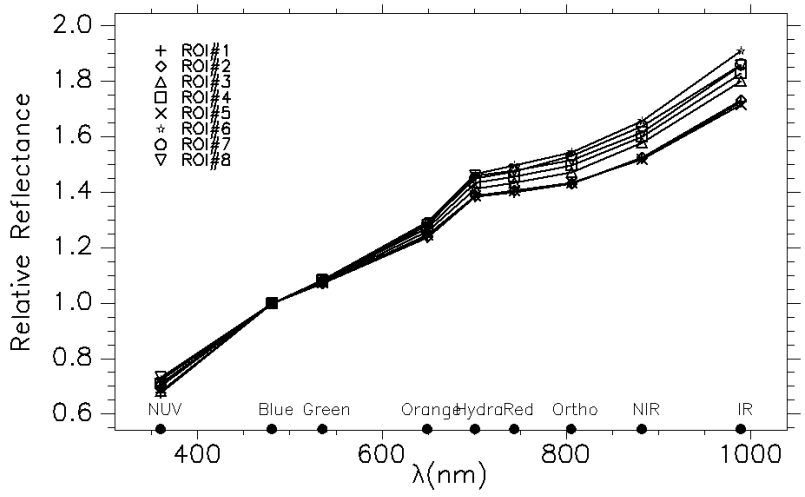

(b) Relative reflectance spectra of ROIs.

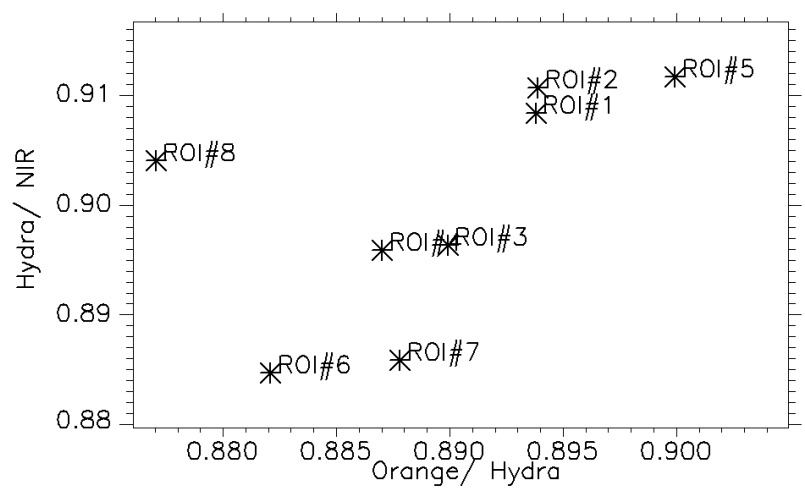

(c) Spectral ratios.

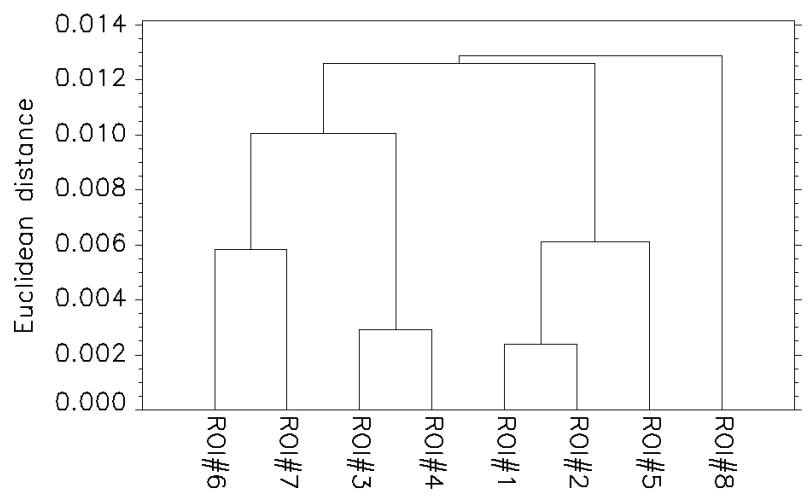

(d) Dendrogram of hierarchically clustered data.

Fig. 4. Spectral properties of defined ROIs in Fig. 3c. Symbols representing ROIs are given in the labels together with the filter names given with bullets located at their central wavelength positions. 
A\&A 586, A80 (2016)

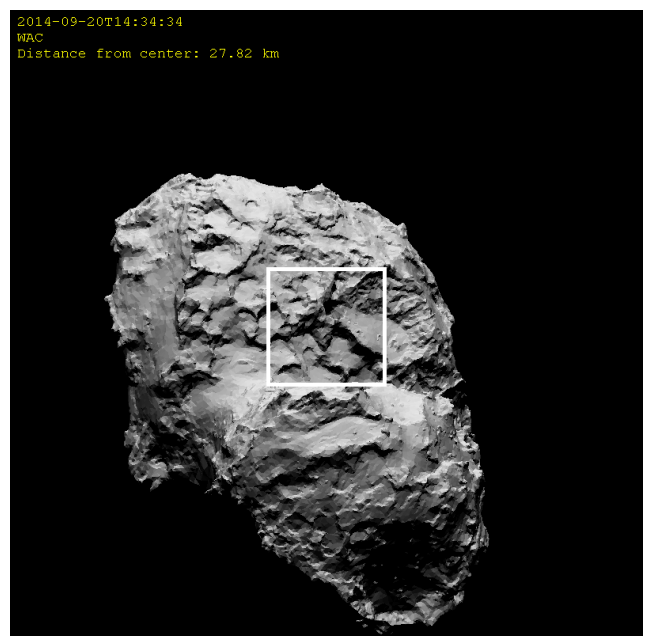

(a) WAC view of image \#3 with the NAC field of view indicated with a white square.

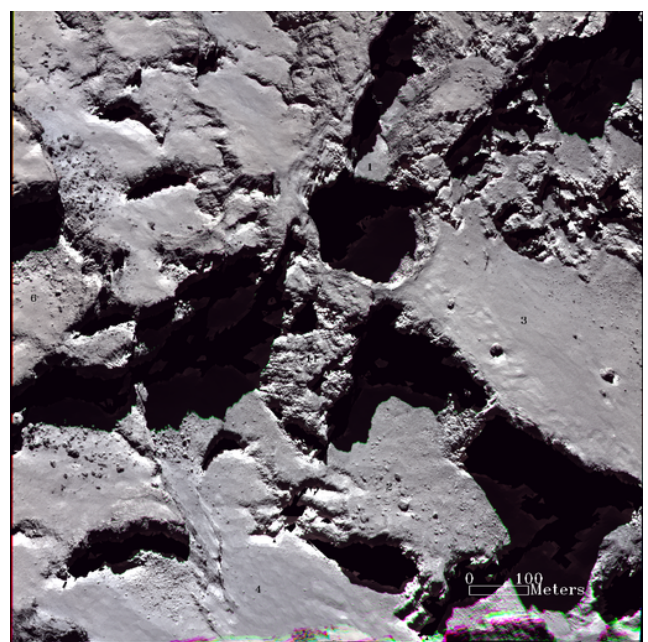

(b) Seth region in image \#3. R: NIR, G: Orange, B: Blue.

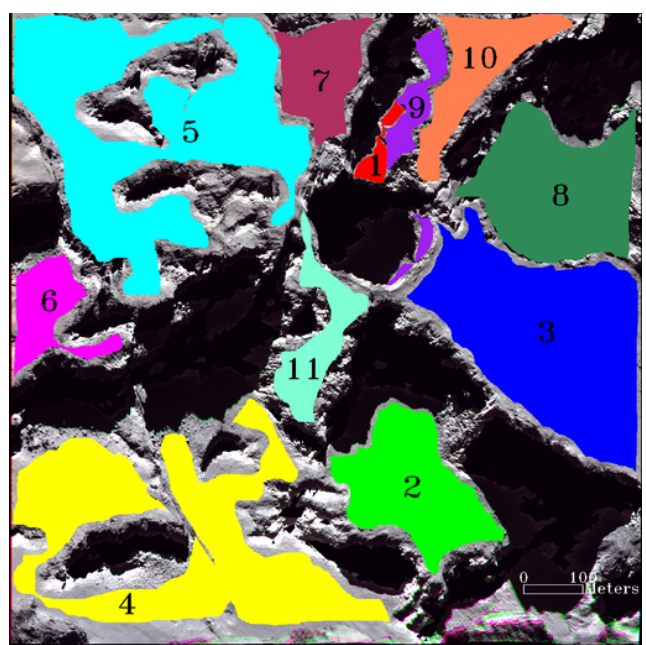

(c) Same RGB images as Fig. 5b, with region of interests overlaid.

Fig. 5. View of the image \#3 (top panel). RGB colors of Seth pits and their surrounding (middle panel) with defined region of interests (bottom panel). Image details can be found with corresponding image ID in Table 2.

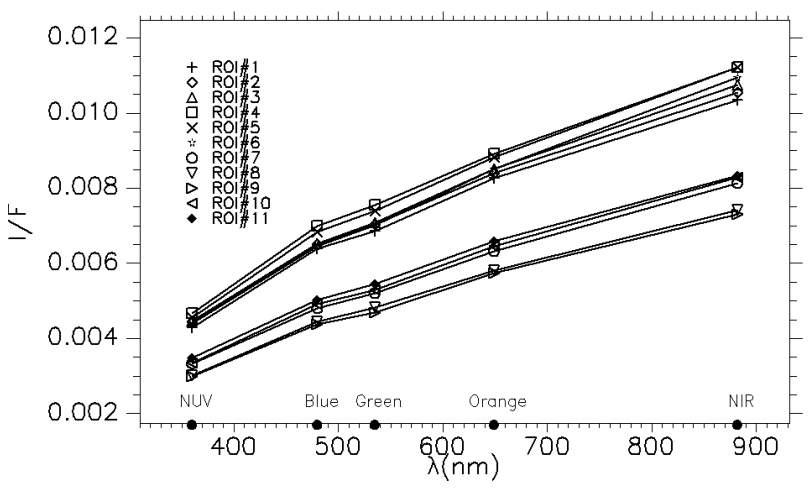

(a) Mean reflectance spectra of ROIs. Typical standard deviation of the data points from the resistant mean is $7.0 \times 10^{-6}$.

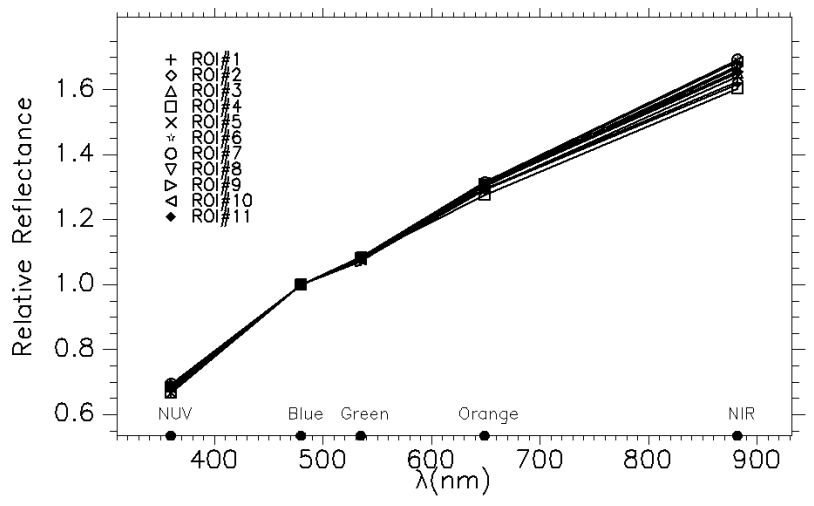

(b) Relative reflectance spectra of ROIs.

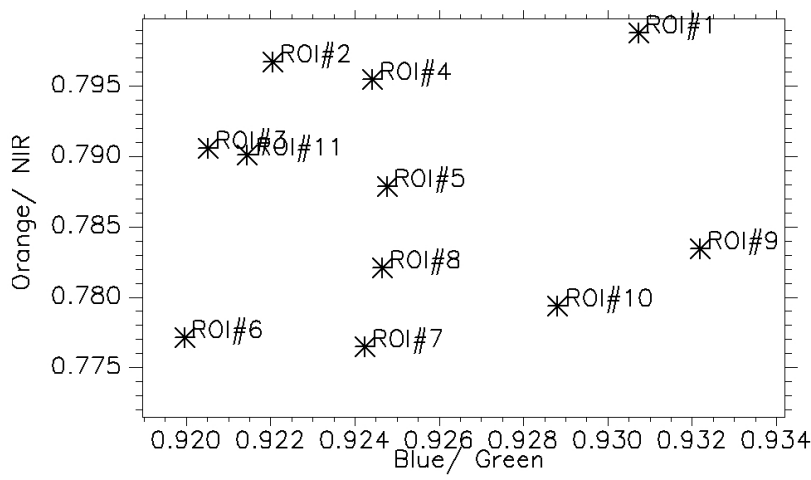

(c) Spectral ratios.

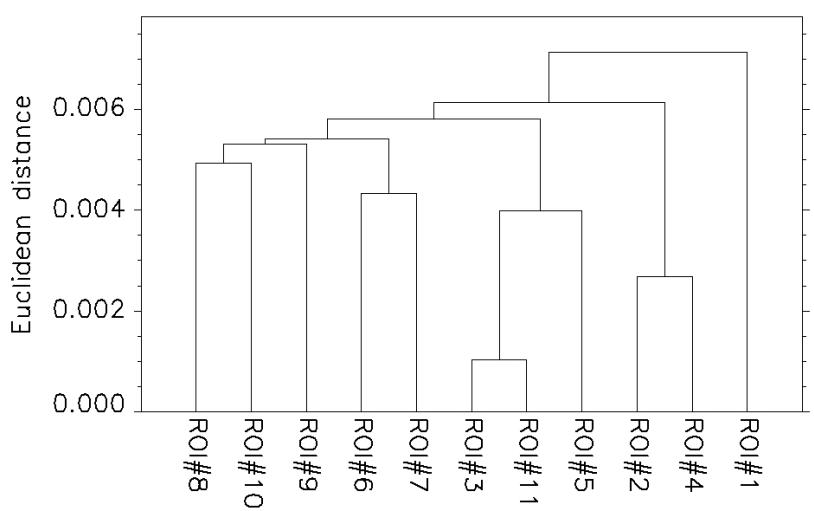

(d) Dendrogram of hierarchically clustered data.

Fig. 6. Spectral properties of ROIs. Symbols representing ROIs are given in the labels together with the filter names given with bullets located at their central wavelength positions. 
According to the results of the clustering analysis, there are similarities between ROIs \#6 and \#7 and ROIs \#8 and \#10 (Fig. 6d) but similarities seem to be related to the morphology of the region.

\subsection{Ma'at region}

The Ma'at region, located on the head of the comet, hosts four pits, and jets were detected in two of them in September 2014. See Fig. $2 b$ for an example of a jet detected in one of the Ma'at pits. As the comet gets closer to the Sun, most pits and alcoves in this area have also shown signs of activity (Vincent et al. 2015, 2016). In the image series we investigated, only three of the pits are visible including the two showing activity (see Fig. B.3, panel c). The WAC view for the image \#2 (details in Table 2) is given in Fig. 7a with the NAC FOV indicated in a white rectangle as a reference. The Ma' at pits and their vicinity are presented in RGB colors using the images taken in filters NIR, orange and blue, respectively, in Fig. 7b, together with a small portion of the Hapi region (top left blue part on the image, indicated in Fig. B.3, panel c). Places where we see activity (pits and Hapi) show bluer color than their vicinity. Selected ROIs are shown in Fig. 7c with their sizes given in Table C.3. The mean reflectance of the ROIs are calculated and presented in Fig. 8a. The spectra of the ROIs have a red slope and are featureless. In the relative reflectance (normalized at $480.7 \mathrm{~nm}$ ) plots given in Fig. 8b, there is a separation between the ROIs \#1, \#9, and \#10 (Ma'at pits) and the rest of the ROIs because of the bluer spectra of the active regions.

Similar to the high resolution images of the Seth region investigated in Sect. 3.2, we have a limited number of filters in this set of Ma'at images. For consistency, the same spectral ratio plot of blue/green and orange/NIR are applied to the mean spectra for the investigation of selected ROIs. In the spectral ratio plot shown in Fig. 8c, two main clusters are clearly detected: ROIs \#1, \#9, \#10 (active pits) and the rest of the ROIs. The clustering analysis applied to the data shown in the spectral ratio plot (Fig. 8c) are presented using dendrogram in Fig. 8d. The clustering analysis shows the existence of four classes:

1. ROIs \#1, \#9, \#10 Ma'at pits (active regions);

2. ROIs \#2, \#5 (one type of mixed regions, where the active and inactive material is probably well mixed);

3. ROIs \#3, \#4, \#6, \#8 (another type of mixed region in which mainly inactive surface material is observed); and

4. ROI \#7 (inactive surface).

\subsection{Imhotep region}

The Imhotep region, located on the body (larger lobe) of the comet, hosts many bright features, which were investigated by Pommerol et al. (2015b). The region has a bright edged basin and many roundish features with dark edges and is surrounded by bright material (Auger et al. 2015). Examples of these features are shown in Fig. B.3, panel d.

The simulated WAC view of the image \#4 (details in Table 2) is shown as a reference with NAC FOV denoted with a white rectangle. Both isolated and clustered bright features (see Fig. B.4 for definitions) are very pronounced with their bright blue colors, even in basic RGB image shown in Fig. 9b. Bright material around the roundish features appears to be greener in this RGB scheme. The rest of the surface is more or less in uniform gray-brown color. Defined ROIs are shown (Fig. 9c) with their sizes listed in Table C.4. Mean reflectance spectra of each ROI are calculated and presented in Fig. 10a. IBFs, selected as
ROI \#4 (IBF1) and ROI \#5 (IBF2), have very flat spectra, while the other ROIs display red slopes with spectral features similar to those seen in the other investigated regions (see Sect. 3.2 for comparison). ROI \#4 is significantly brighter than the other ROIs and it displays a potential absorption feature at $700 \mathrm{~nm}$, while the mean spectra of the other ROIs seem to be affected by the cometary emission in the $700-750 \mathrm{~nm}$ range, as discussed in Sect. 3.2. In the relative reflectance (normalized at $480.7 \mathrm{~nm}$ ), the spectral shapes of the ROIs are more clear and the comparison of ROIs can be carried out more easily. The spectra of IBFs (ROIs \#4, \#5) have the bluest spectra and clustered bright features (ROIs \#1-\#3) have bluer spectra than the rest of the ROIs. Spectral ratio plots were prepared using the same filters as those used in the investigation of the Seth and Hapi regions (image \#1, Table 2), since they were found to be the best for this ROI analysis. Orange/hydra and hydra/NIR ratio plots are presented in Fig. 10c. IBF1, showing a potential $700 \mathrm{~nm}$ absorption band (ROI \#4), is well separated from the rest of ROIs with its high ratio values. Similarly the IBF2, which has a very flat spectrum (ROI \#5), has high ratio values and is well separated from the rest of the ROIs. The clustered bright features (ROIs \#1, \#2, \#3) are grouped together. Their high ratio values indicates that they are active, but there was no activity detected from those clustered bright features until 12 April 2015 (see Fig. 2c). Nevertheless, water ice was detected by the Visible and InfraRed Thermal Imaging Spectrometer-Mapper (VIRTIS-M) in the NIR region (Filacchione et al. 2015). The ratio values of these clustered bright features are similar to those of Seth pits and Hapi, while the ratios of IBFs are much higher. The rest of the ROIs are clustered together except ROI \#11, which is classified as an inactive surface.

The clustering analysis applied to the data displayed in the spectral ratio plots (Fig. 10c) is presented using dendrogram in Fig. 10d. This analysis indicates five main groups of ROIs:

1. ROI \#4: Isolated bright feature (IBF1);

2. ROI \#5: Isolated bright feature (IBF2);

3. ROIs \#1, \#2, \#3: Clustered bright features (active regions);

4. ROI \#11: Inactive surface; and

5. ROIs \#6, \#7, \#8, \#9, \#10: Mixed regions.

According the clustering analysis, active surface is in three different groups: 1. ROI \#4 (IBF1), 2. ROI \#5 (IBF2), and 3. ROIs \#1, \#2, \#3 (clustered bright features). ROI \#11 is the inactive surface represented by the lowest spectral ratio values. The rest of the ROIs represent the mixed surface. The mixed region could be investigated in four classes: 1 . ROIs \#6, \#8, 2. ROI \#12, 3. ROIs \#7, \#9, and 4. ROI \#10.

Once the ROIs of the mixed regions are investigated separately taking the spectral slopes and the decorrelation stretched images of these regions into account, we concluded that these regions must contain regions showing activity due to their lower spectral slopes and the colors closer to the regions where the activity was detected. This result is supported by the stereoscopic reconstruction of the source region of an outburst observed on 12 March 2015 rising from the Imhotep region (Knollenberg et al. 2015). The source region of the outburst was determined as the rough terrain, where the roundish features are, on Imhotep. The detailed spectral analysis of the roundish features and their surrounding can be found in Knollenberg et al. (2015).

Since the most plausible explanation for the bright features on the surface of comet 67P is that they are icy patches (Pommerol et al. 2015b), and therefore possible sources of activity, it is strange that we did not observe any activity rising from IBF1 and IBF2. 
A\&A 586, A80 (2016)

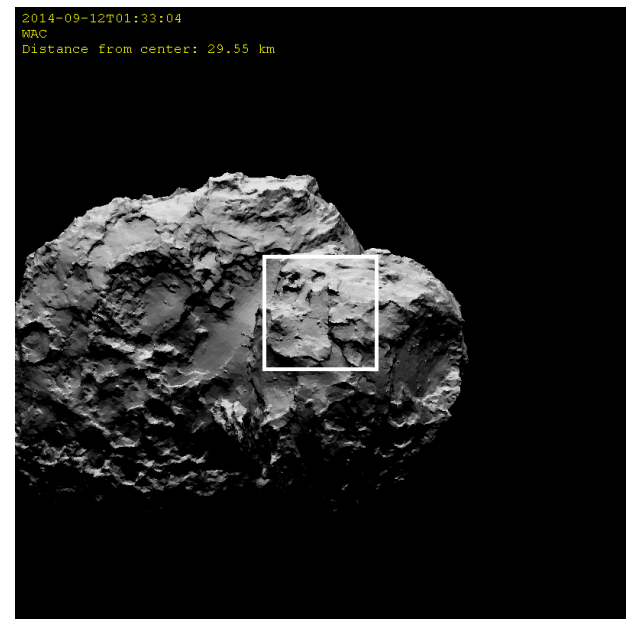

(a) WAC view of the image \#2 with the NAC field of view indicated with a white square.

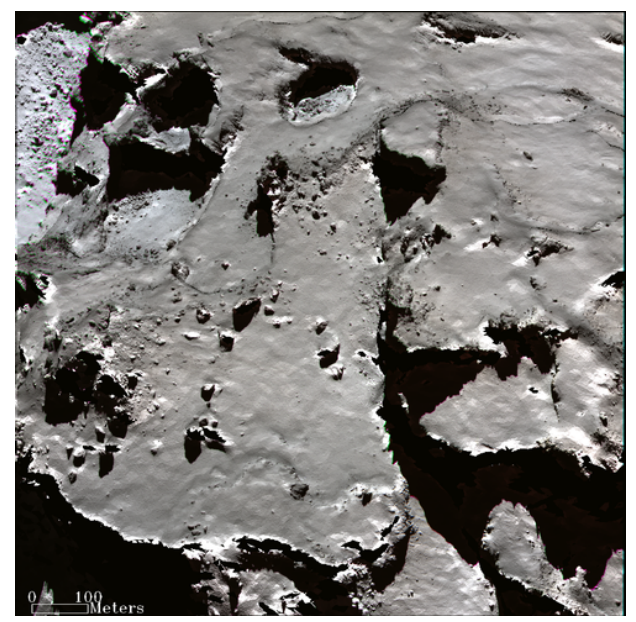

(b) Ma'at region in image \#2. R: NIR, G: Orange, B: Blue.

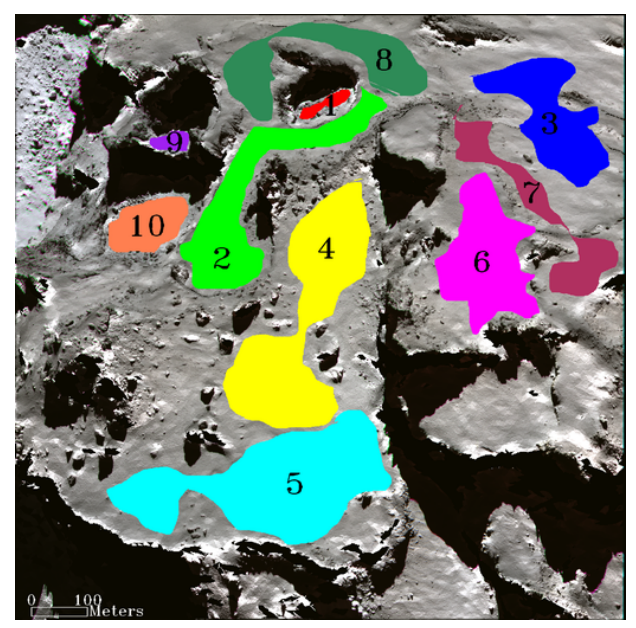

(c) Same RGB images as Fig. 7b, with region of interests overlaid.

Fig. 7. View of the image \#2 (top panel). RGB colors of Ma'at pits and their surrounding (middle panel) with defined region of interests (bottom panel). Image details can be found with corresponding image ID in Table 2.

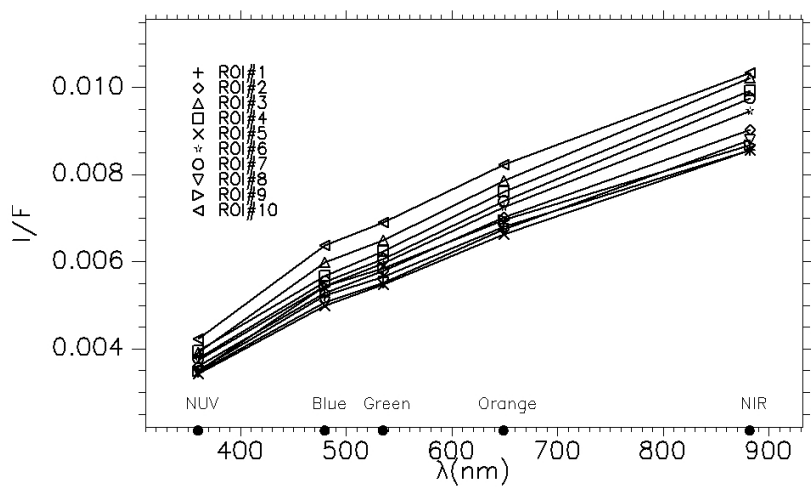

(a) Mean reflectance spectra of ROIs. Typical standard deviation of the data points from the resistant mean is $7.0 \times 10^{-6}$.

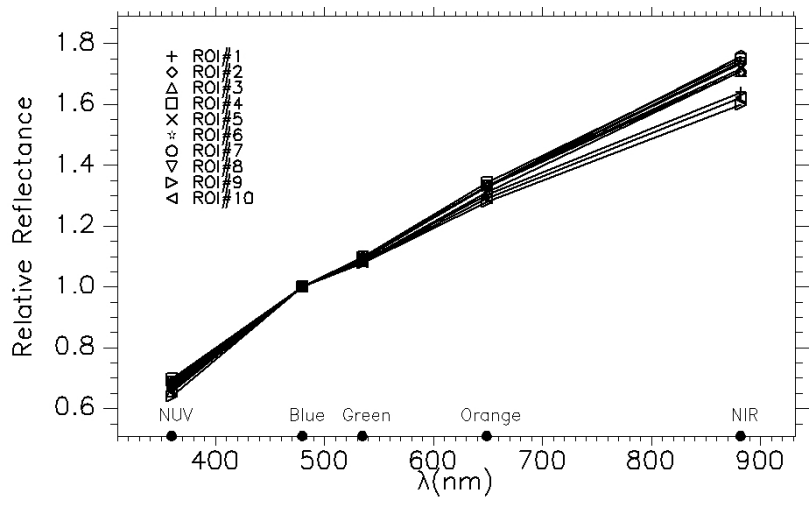

(b) Relative reflectance spectra of ROIs.

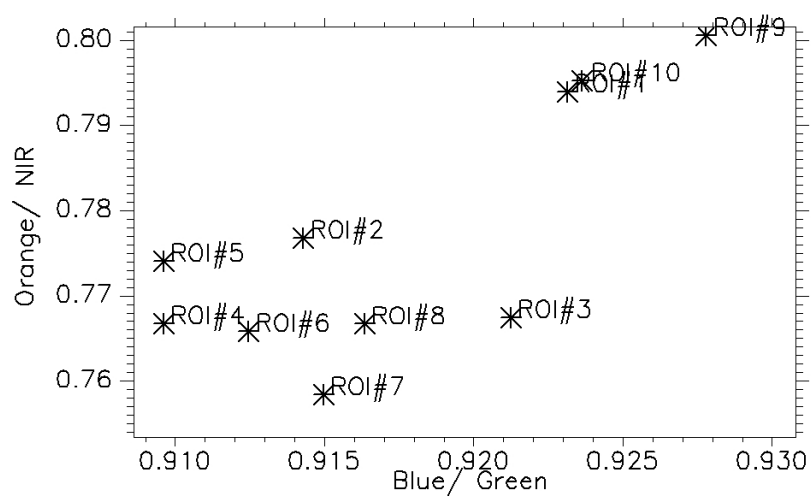

(c) Spectral ratios.

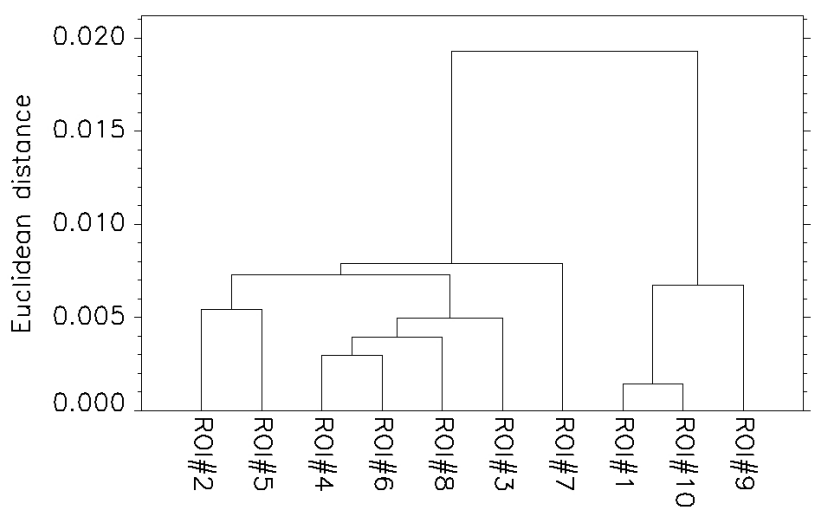

(d) Dendrogram of hierarchically clustered data.

Fig. 8. Spectral properties of ROIs. Symbols representing ROIs are given in the labels together with the filter names given with bullets located at their central wavelength positions. 
N. Oklay et al.: Variegation of comet 67P's active regions

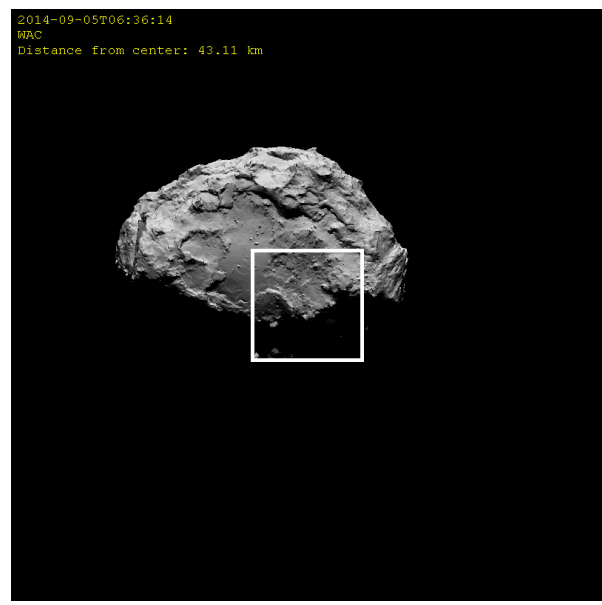

(a) WAC view of the image \#4 with the NAC field of view indicated with a white square.

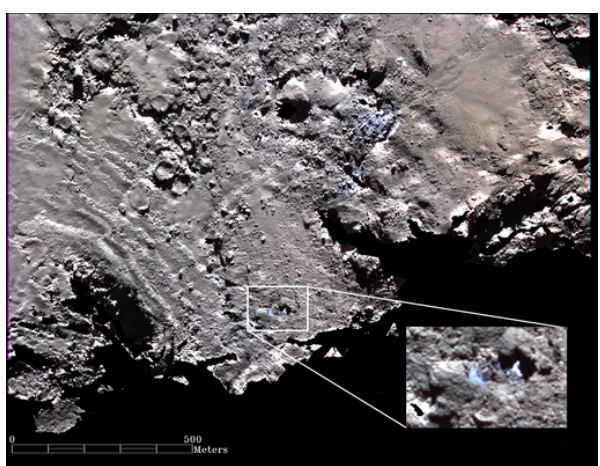

(b) Imhotep region in image \#4. R: NIR, G: Orange, B: Blue.

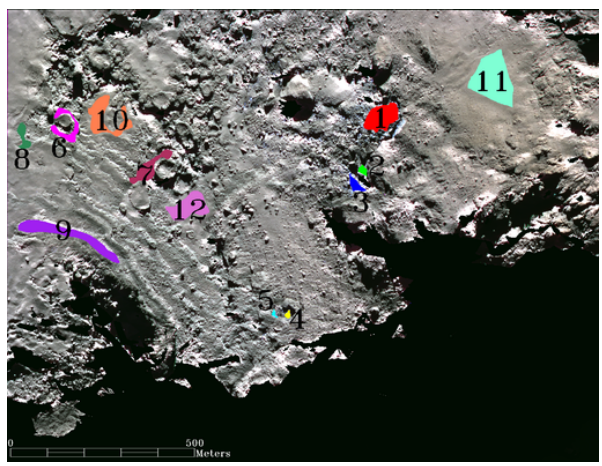

(c) Same RGB images as Fig. 9b, with region of interests overlaid.

Fig. 9. View of the image \#4 (top panel). RGB colors of Imhotep active regions and their surrounding (middle panel) with defined region of interests (bottom panel). Image details can be found with corresponding image ID in Table 2.

\section{Discussions}

Spectral ratio analysis is a very powerful technique to detect active regions on the surface of comet 67P. Using the lower limits determined by the spectral ratio plots presented in the Sect. 3, the activity thresholds listed in Table 3 are introduced and used in the detection of potential active regions in the investigated images.

The results obtained by applying the activity thresholds method is shown in Fig. 12. Light colors (white, light blue and light pink) correctly indicate the regions in which activity was

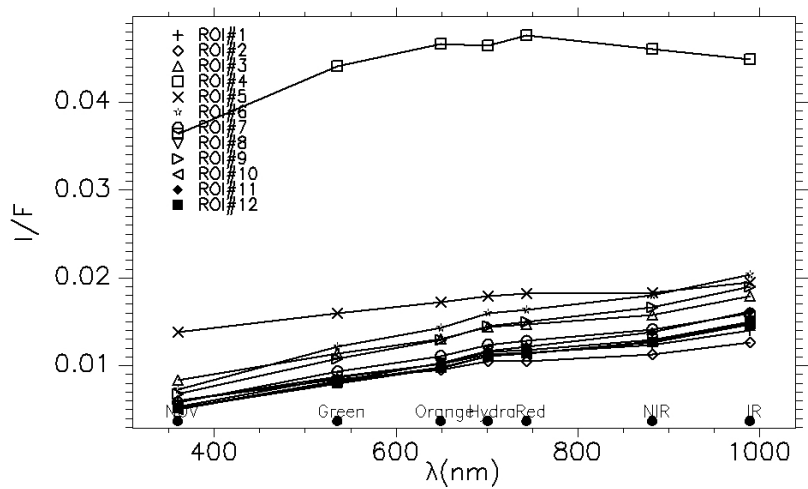

(a) Mean reflectance spectra of ROIs. Typical standard deviation of the data points from the resistant mean is $2.3 \times 10^{-4}$.

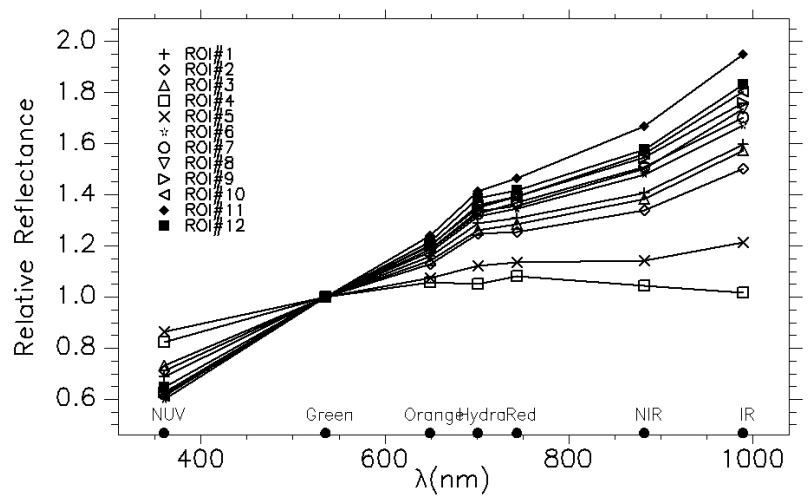

(b) Relative reflectance spectra of ROIs.

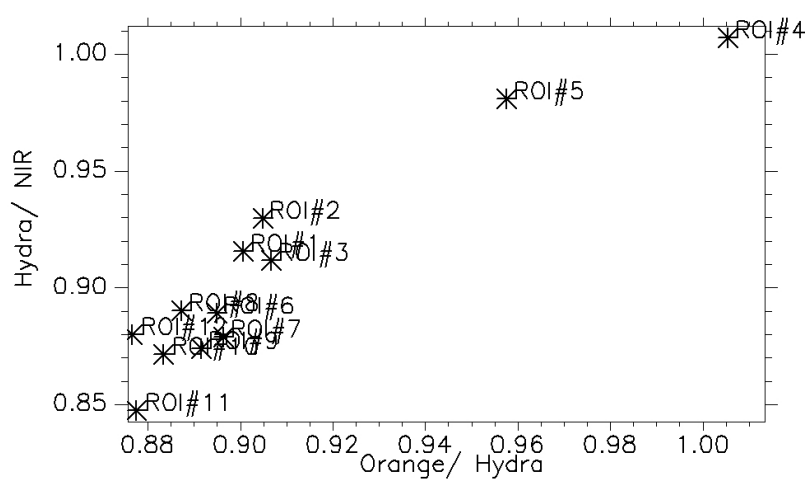

(c) Spectral ratios.

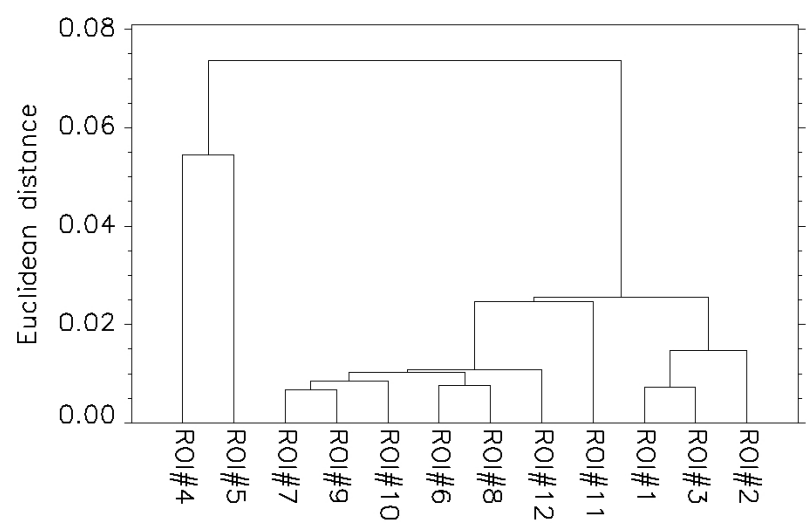

(d) Dendrogram of hierarchically clustered data.

Fig. 10. Spectral properties of ROIs. Symbols representing ROIs are given in the labels together with the filter names given with bullets located at their central wavelength positions. 


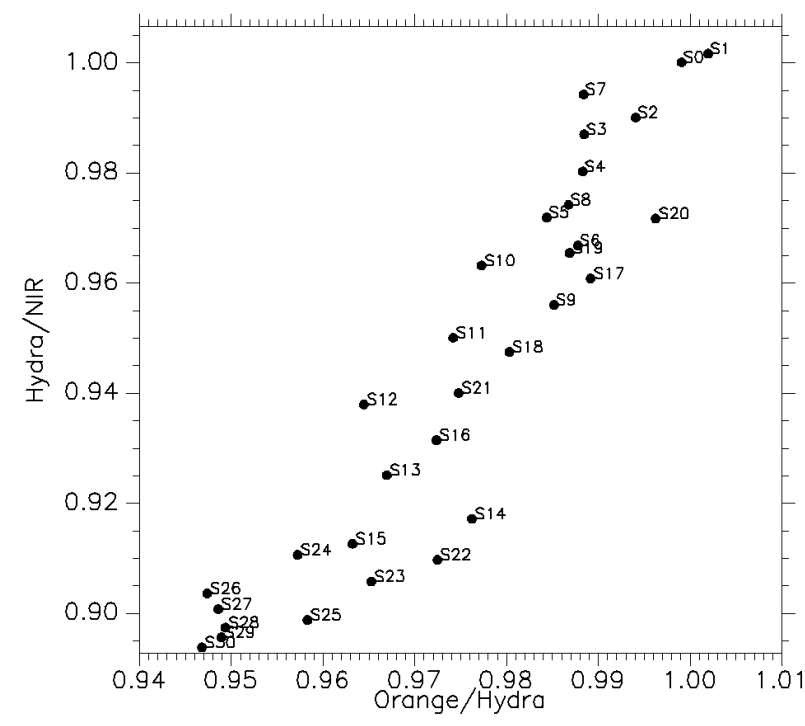

Fig. 11. Ratios of icy mixture of laboratory data. S0 is the spectrum recorded at $t=0.33 \mathrm{~h}$, while S30 is the spectrum recorded ar $t=$ $30.35 \mathrm{~h}$. The aged mixture between S26 $(t=26 \mathrm{~h})$ and $\mathrm{S} 30(t=30.35 \mathrm{~h})$ have values between $0.945-0.95$ in orange-hydra ratio while the values are between $0.89-0.905$ in hydra-NIR ratio.

Table 3. Activity thresholds for two types of observations according to the availability of images taken in hydra filter.

\begin{tabular}{lcc}
\hline \hline Channels & $\begin{array}{c}\text { Hydra } \\
\text { available }\end{array}$ & $\begin{array}{c}\text { Hydra not } \\
\text { available }\end{array}$ \\
\hline $\mathrm{R}$ & (Hydra/NIR $) \geq 0.908$ & (Orange/NIR) $\geq 0.794$ \\
$\mathrm{G}$ & Orange & Orange \\
$\mathrm{B}$ & (Orange/Hydra) $\geq 0.893$ & $($ Blue/Green $) \geq 0.922$ \\
\hline
\end{tabular}

Notes. The best detection of active regions was achieved using the images taken with orange, hydra, and NIR filters but because operational constrains, not all observing sequences include all filters. Therefore, another definition is introduced for a common observing sequence.

detected (Hapi, Seth pits, Ma' at pits) but also some other regions (large Anuket alcove, alcoves in Seth and Ma'at, clustered BFs, IBFs and around roundish features in Imhotep), where the activity was not visually detected at the time of observations probably owing to limited observation conditions. Activity from some of those features (Seth and Anuket alcoves) were actually indirectly detected via the inversion of dust jets (Vincent et al. 2016) and stereoscopic reconstruction of the outburst observed on 12 March 2015, which originated from the roundish features of Imhotep (Knollenberg et al. 2015). Additionally, jets rising from the clustered bright features of Imhotep were also observed on 12 April 2015 (see Fig. 2c). Therefore, even if we did not have direct observations to state that all the potential active regions detected by the activity thresholds method are really active, the following observations of the investigated regions, along with indirect observations, proved that this is a highly reliable method to determine the potentially active regions on the comet surface.

As a result of the various filter combinations used during the observations, the comparison of the images used in this study could only be carried out with four filters (F16, F23, F22, F41). In this situation, the ratios of near-UV/green and orange/NIR are found to be the best suited for this purpose. In Fig. 13 it is seen that the ROIs\#4 and \#5 of the Imhotep region (IBFs) are well separated from the rest of the ROIs and they fall on the high ratio-ratio region. ROIs \#1, \#2, \#3 (clustered bright features) on the Imhotep region and ROIs \#1, \#2, \#5 (Seth pits and Hapi) of the image \#1 are also grouped together and well separated from the rest of the ROIs. All the regions above 0.79 in the orange/NIR ratios seem to represent active and potential active surface with the exception of ROI\#3 of Seth on image \#1 and ROI\#6 of Imhotep region. The first exceptional regions happened to be those covered by the deposited material and the ratios used are not able to make a reliable distinction among them. The second was already predicted to be active (see Sect. 3.4 for the discussion) and the source of the outburst seen about a half year after is this region (Knollenberg et al. 2015). No separation of inactive and mixed surface regions could be achieved using only four common filters (see Fig. 13). Nevertheless the regions displaying activity can be separated from the rest of the surface together with the isolated bright features, which were suspected to be active.

To investigate these IBFs in more details, the spectral ratio plots of laboratory data of icy mixtures (see Sect. 2.4) have been calculated with the same filters (orange, hydra, NIR) and the same ratios (orange/hydra, hydra/NIR) as the investigated data with hydra filter. Spectral ratio plot of the laboratory data are presented in Fig. 11. The ratios calculated from the spectra of the icy mixture in time show a decrease along both axes in time. The lower limit after about $30.35 \mathrm{~h}$ is 0.946 in orange/hydra, while it is 0.895 in hydra/NIR. Those values are a bit lower ( 0.893 in orange/hydra and 0.908 in hydra/NIR) for the general active regions, however, the spectral ratio values of both IBF1 and IBF2 are in the range of the ratios of laboratory spectra. This result suggests that the investigated laboratory mixture is representative for the material observed on the comet, supporting the previous work by Pommerol et al. (2015b), who investigated the bright points. These authors found $\mathrm{H}_{2} \mathrm{O}$ ice to be the most reasonable explanation for bright features on the comet surface. This is finally confirmed by the VIRTIS spectrometer observations in the NIR range (Filacchione et al. 2015) for at least two clustered bright features, including the one studied in this work. According to this comparison, these regions might contain ice with similar mixtures to those used in the laboratory experiments. IBF2 diverges from the main cluster of the laboratory data, which might indicate a deviation from the mixture used the laboratory. IBF1 falls close to the fresh ice and dust laboratory mixture before sublimation of volatile component, indicating that it has been exposed to the surface not long ago.

By combining all results presented in Sect. 3, the surface regions of comet $67 \mathrm{P}$ can be classified into three main categories:

1. Regions of low spectral slopes $(8-13 \% / 100 \mathrm{~nm})$ are correlated with sources of activity: active pits, alcoves, bright features. These regions are located in the high ratio regions in the spectral ratio plots and are blue in decorrelation stretched images.

2. Regions of high spectral slopes (above 15\%/100 nm) are correlated with inactive surfaces. These regions are located in the low ratio regions in the spectral ratio plots and are orange in decorrelation stretched images.

3. Regions of intermediate spectral slopes $(13-15 \% / 100 \mathrm{~nm})$ correspond to either surfaces partially covered by material ejected from active spots or inactive areas peppered with small bright spots, which are currently interpreted as minor ice deposits (Pommerol et al. 2015b). These regions are located in the intermediate ratio regions in the spectral ratio plots and are light orange and green. 


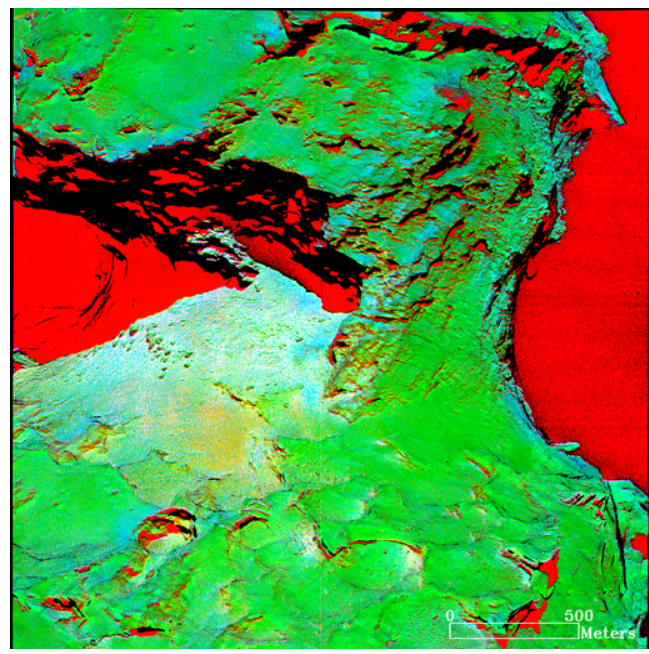

(a) Seth, image \#1, R: Hydra/NIR $\geq 0.908$, G: Orange, B: Orange/Hydra $\geq 0.893$.

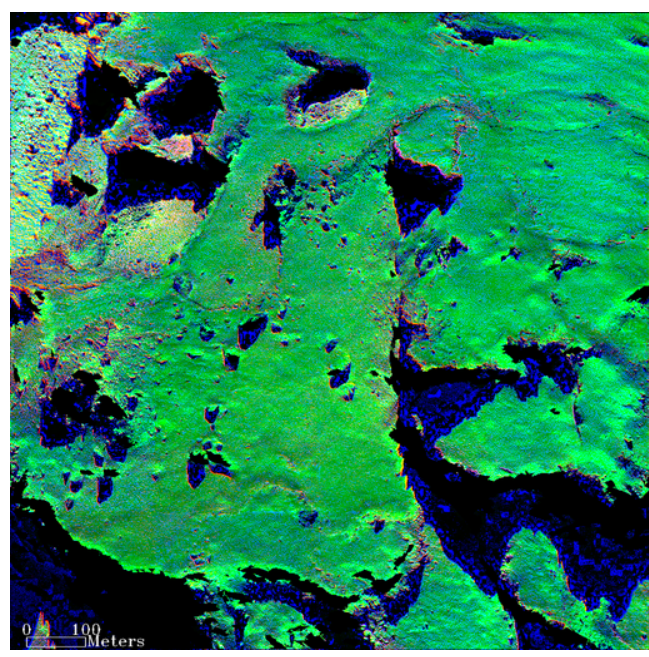

(c) Ma'at, image \#2, R: Orange/NIR $\geq 0.794, \mathrm{G}$ : Orange, B: Blue/Green $\geq 0.922$.

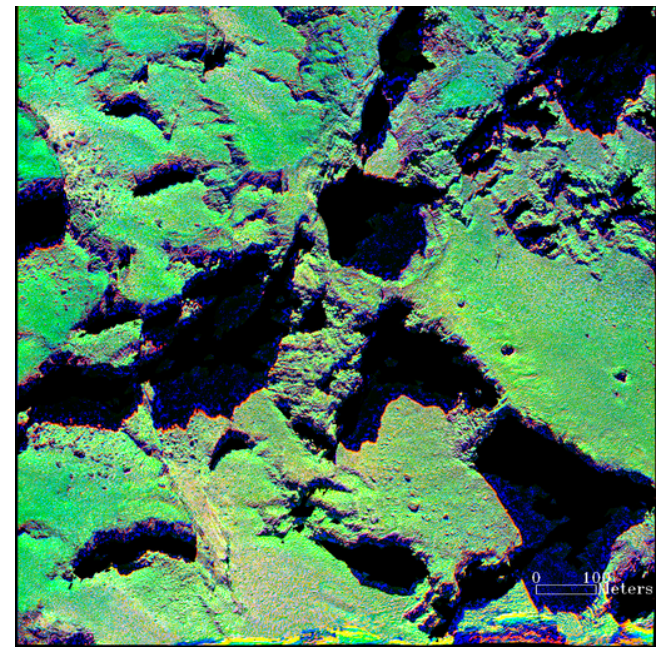

(b) Seth, image \#3, R: Orange/NIR $\geq 0.794$, G: Orange, B: Blue/Green $\geq 0.922$.

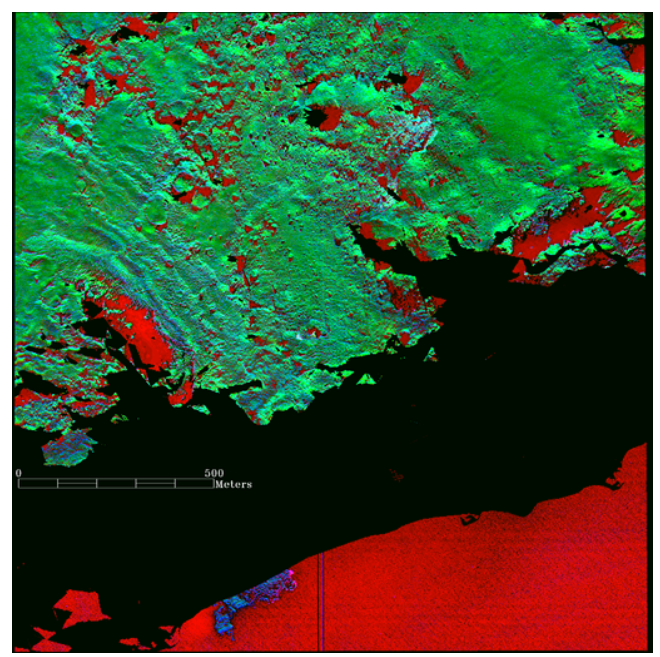

(d) Imhotep, image \#4, R: Hydra/NIR $\geq 0.908$, G: Orange, B: Orange/Hydra $\geq 0.893$.

Fig. 12. Activity thresholds (Table 3) determined by the spectral ratio plots and assigned to RGB channels. In the images, black indicates the regions excluded because of high geometric angles, while red denotes the low signal regions like shadows and the sky. The shadows, where we still receive decent signals, are shown in shades of blue. Regions where we see activity are in light blue and light pink. Using such images, we can detect the active regions. Image details can be found with corresponding image ID in Table 2.

Three groups of terrains defined based on the global spectral slopes of the comet (Fornasier et al. 2015) are thus extended using activity thresholds and refined in this way for the small scale surface regions as active, inactive, and mixed surface with high resolution images of the comet.

All spectra investigated display a red slope. The regions showing activity have bluer slopes, which we interpret as due to the presence of water ice on the basis of the icy laboratory experiments on icy samples (see Sect. 3.4 and Pommerol et al. 2015b), and on the basis of the correlation between exposed water ice deposits and bluer spectral slopes found on comet 9P/Tempel 1 Sunshine et al. (2006) and comet 103P/Hartley 2 (Li et al. 2013). Detection of water ice by VIRTIS-M on the clustered bright feature we studied (Filacchione et al. 2015) supports our interpretation for the bluer spectral regions we investigated.

The slope change between NIR and IR seen in Fig. 4b and in Fig. 10b indicates a faint absorption feature between
800-900 nm, but the coma contribution between the camera and the comet's surface prevents further spectral feature detection.

An absorption band around $700 \mathrm{~nm}$ is potentially detected in the spectrum of IBF1. The negative reddening values of the dust grains may also suggest such an absorption band (Cremonese et al. 2016). A possible explanation for the potential $700 \mathrm{~nm}$ absorption feature is that some bright boulders contain hydrated minerals such as phyllosilicates. Formation of phyllosilicates requires that anhydrous minerals, such as olivine and pyroxene, have been exposed to liquid water. Such aqueous alteration is common in carbonaceous chondrites (e.g., Rubin 1997). Heating of icy planetesimals to the point of water ice melting, e.g., by Al-26 decay, leads to loss of supervolatiles and porosity (Prialnik et al. 2008). When these compacted bodies refreeze, the tensile strength may reach several MPa.

Davidsson et al. (2015) therefore argued that the low bulk densities, high porosities, weak strengths, and abundance of 
A\&A 586, A80 (2016)

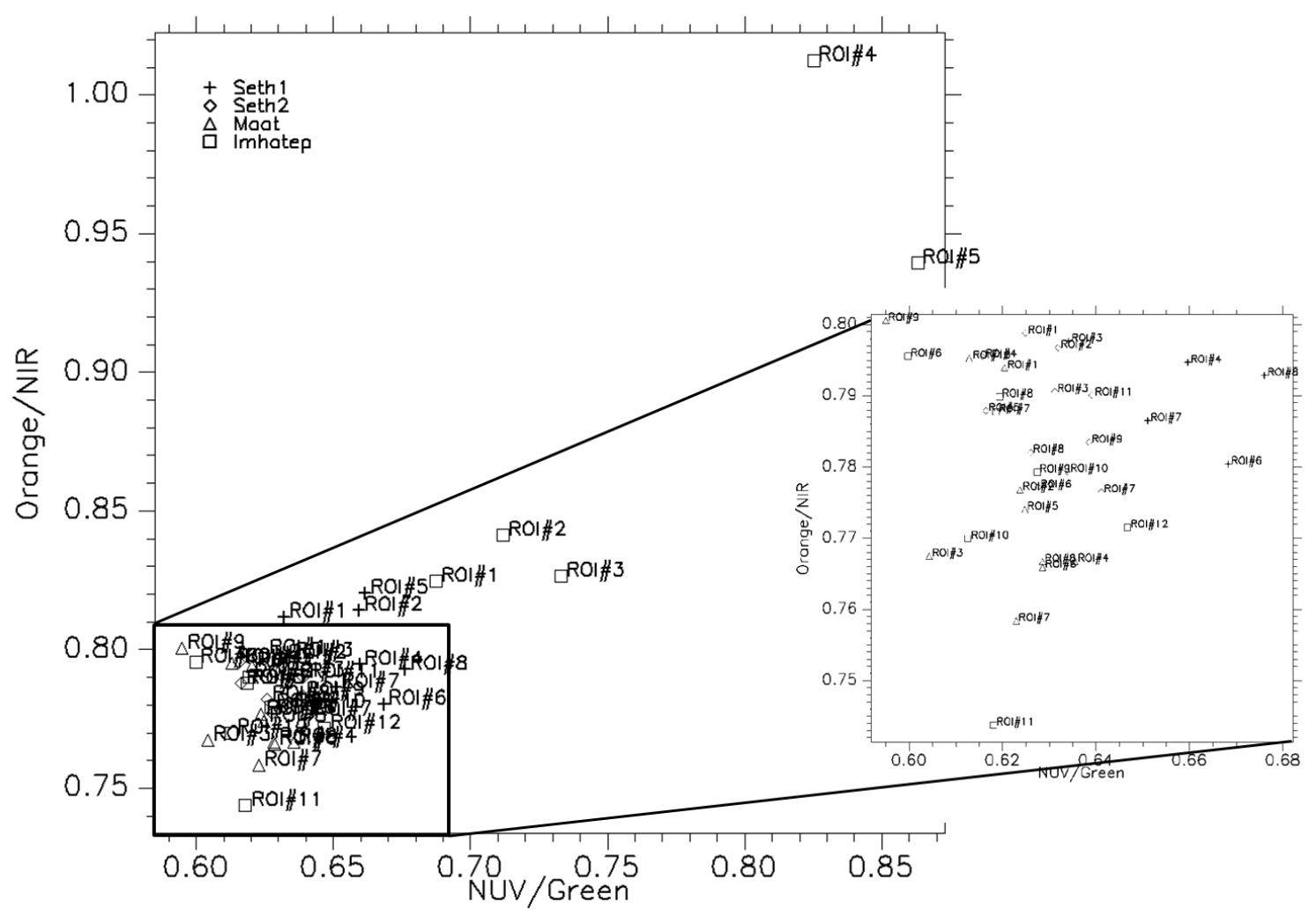

Fig. 13. Spectral ratios of each ROIs of all sets of images in four common filters.

supervolatiles in comet nuclei excludes in situ water melting in these bodies. In fact, they used the lack of measurable $700 \mathrm{~nm}$ absorption for the vast majority of the 67P nucleus surface to support that claim. In their view, comets form by hierarchical agglomeration (Weidenschilling 1997) and the combination of small initial nucleus sizes and slow growth efficiently prevents thermal processing by Al-26. Davidsson et al. (2015) also argued that most large $(D>50 \mathrm{~km})$ bodies in the primordial disk constitute a population distinctly different from comets, which formed early at large initial sizes through streaming instabilities (Johansen et al. 2007). They used the captured saturnian satellite Phoebe as an example of how strong thermal processing in this population led to significant compaction and aqueous alteration, revealed by a high bulk density and clear $700 \mathrm{~nm}$ absorption.

If this scenario is correct, a possible interpretation of our observation is that some bright boulders on 67P do not constitute indigenous comet material. Although collisions among large thermally processed bodies may have been very inefficient producers of escaping ejecta (Davidsson et al. 2015), it is not unlikely that a population of small $(\leq 10 \mathrm{~m})$ phyllosilicate-rich fragments built up in the primordial disk over time. If so, comet nuclei may have swept up this foreign debris material during their growth. The fact that the bright boulders differ significantly in terms of albedo and spectral properties with respect to the rest of the comet surface, combined with their presence in several different types of morphological environments on the comet, might support this unusual origin. Further possible support for this scenario is that one out of five studied piles of bright boulders on 67P has a size distribution that suggests break-up during impact rather than gravity-driven fragmentation during masswasting (Pommerol et al. 2015b). If some of the bright boulders are so-called strangers, they may provide important information about other populations of bodies present in the environment in which comets formed.

\section{Conclusions and outlook}

The multispectral OSIRIS NAC data plays an essential role in the detection of the surface inhomogeneities and activity. Activity thresholds introduced in this work provide an independent method to identify both currently active regions and potential active regions, even in the observing sequences using limited number of filters. In most of the regions detected as potentially active in the activity threshold analysis, the activity was detected in spring 2015 either visually or via inversion methods. This late detection might be because of the limited observing conditions or the lack of energy input for activity to start. We found that the three filters, orange, hydra, NIR, provide useful color ratios for the identification of active, inactive, and mixed surface regions.

Spectral ratio analysis indicates two different types of active regions: 1. Isolated bright features in Imhotep region. 2. Hapi, active pits, alcoves, clustered bright features. Two types of mixed regions are detected in the spectral ratio analysis: 1. Regions containing both active and inactive surface features. One example of such a region is the one around Seth pits containing alcoves; another example is the large alcove of Anuket, which seems to contain both active and inactive material. 2. Regions containing material that was lifted up due to comet activity, fall down on the comet surface and mix with the material where it landed. We mostly see these regions around pits, around roundish features, and around Hapi. Surface deposits presented in Thomas et al. (2015a) are consistent with the mixed regions detected in this study.

A potential absorption feature $700 \mathrm{~nm}$ is detected in IBF1. This absorption line may be attributed to hydrated minerals (Vilas 1994).

Acknowledgements. OSIRIS was built by a consortium of the Max-PlanckInstitut für Sonnensystemforschung, Göttingen, Germany, CISAS University 
of Padova, Italy, the Laboratoire d'Astrophysique de Marseille, France, the Institutode Astrofísica de Andalucia, CSIC, Granada, Spain,the Research and Scientific Support Department of the European Space Agency, Noordwijk, The Netherlands, the Instituto Nacionalde Técnica Aeroespacial, Madrid, Spain, the Universidad Politéchnica de Madrid, Spain, the Department of Physics and Astronomy of Uppsala University, Sweden, and the Institut für Datentechnik und Kommunikationsnetze der Technischen Universität Braunschweig, Germany. The support of the national funding agencies of Germany (DLR), France(CNES), Italy(ASI), Spain(MEC), Sweden(SNSB), and the ESA Technical Directorate is gratefully acknowledged. We thank the Rosetta Science Ground Segment at ESAC, the Rosetta Mission Operations Centre at ESOC, and the Rosetta Project at ESTEC for their outstanding work enabling the science return of the Rosetta Mission. We thank Cecile Engrand for the discussions. This research has made use of NASA's Astrophysics Data System Bibliographic Services. This research has made use of the USGS Integrated Software for Imagers and Spectrometers (ISIS). We gratefully acknowledge the developers of SPICE and NAIF/PDS resources. We thank Michael J. S. Belton for his comments on the manuscript and suggestions for the improvement of the presented work.

\section{References}

Acton, C. H. 1996, Planet. Space Sci., 44, 65

Anderson, J. A., Sides, S. C., Soltesz, D. L., Sucharski, T. L., \& Becker, K. J. 2004, in Lun. Planet. Sci. Conf., eds. S. Mackwell, \& E. Stansbery, Lunar and Planetary Inst. Technical Report, 35, 2039

Auger, A.-T., Groussin, O., Jorda, L., et al. 2015, A\&A, 583, A35

Bandfield, J. L., Rogers, D., Smith, M. D., \& Christensen, P. R. 2004, J. Geophys. Res. (Planets), 109, 10008

Cremonese, G., Simioni, E. Ragazzoni, R., Bertini, I., et al. 2016, A\&A, accepted Davidsson, B. J. R., Sierks, H. Güttler, C., et al. 2015, A\&A, submitted

El Maarry, M. R., Thomas, N., Preusker, P., et al. 2012, LPI Contributions, 1667, 6404

El-Maarry, M. R., Thomas, N., Giacomini, L., et al. 2015, A\&A, 583, A26

Filacchione, G., De Sanctis, M. C., Capaccioni, F., et al. 2015, Nature, submitted Fornasier, S., Hasselmann, P. H., Barucci, M. A., et al. 2015, A\&A, 583, A30

Gillespie, A. R., Kahle, A. B., \& Walker, R. 1986, Remote Sensing of Environment, 20, 209

Hapke, B. 1993, Theory of Reflectance and Emittance Spectroscopy, 1st edn. (Cambridge University Press)

Johansen, A., Oishi, J. S., Mac Low, M.-M., et al. 2007, Nature, 448, 1022

Keller, H. U., Barbieri, C., Lamy, P., et al. 2007, Space Sci. Rev., 128, 433

Knollenberg, J., Lin, Z.-Y., Hviid, S., et al. 2015, A\&A, submitted

La Forgia, F., Giacomini, L., Lazzarin, M., et al. 2015, A\&A, 583, A41

Li, J.-Y., Besse, S., A'Hearn, M. F., et al. 2013, Icarus, 222, 559

Lin, Z.-Y., Ip, W.-H., Lai, I.-L., et al. 2015, A\&A, 583, A11

Lucchetti, A., Cremonese, G., Jorda, L., et al. 2016, A\&A, 585, L1

Oklay, N., Vincent, J.-B., Sierks, H., et al. 2015, A\&A, 583, A45

Pajola, M., Oklay, N., La Forgia, F., et al. 2015, A\&A, submitted

Pommerol, A., Jost, B., Poch, O., et al. 2015a, Planet. Space Sci., 109, 106

Pommerol, A., Thomas, N., El-Maarry, M. R., et al. 2015b, A\&A, 583, A25

Preusker, F., Scholten, F., Matz, K.-D., et al. 2015, A\&A, 583, A33

Prialnik, D., Sarid, G., Rosenberg, E. D., \& Merk, R. 2008, Space Sci. Rev., 138, 147

Rubin, A. E. 1997, Meteorit. Planet. Sci., 32, 733

Sierks, H., Barbieri, C., Lamy, P., et al. 2015, Science, 347, aaa1044

Sunshine, J. M., A'Hearn, M. F., Groussin, O., et al. 2006, Science, 311, 1453

Thomas, N., Davidsson, B., El-Maarry, M. R., et al. 2015a, A\&A, 583, A17

Thomas, N., Sierks, H., Barbieri, C., et al. 2015b, Science, 347, aaa0440

Tubiana, C., Güttler, C., Kovacs, G., et al. 2015, A\&A, 583, A46

Vilas, F. 1994, Icarus, 111, 456

Vincent, J.-B., Bodewitz, D., Besse, S., et al. 2015, Nature, 523, 63
Vincent, J.-B., Oklay, N., Pajola, M., et al. 2016, A\&A, in press,

DOI: 10.1051/0004-6361/201527159

Weidenschilling, S. J. 1997, Icarus, 127, 290

1 Max-Planck-Institut für Sonnensystemforschung, Justus-vonLiebig-Weg 3, 37077 Göttingen, Germany

e-mail: oklay@mps.mpg.de

2 LESIA, Observatoire de Paris, PSL Research University, CNRS, Sorbonne Universités, Univ. Paris Diderot, Sorbonne Paris Cité, UPMC Univ. Paris 06, 5 place J. Janssen, 92195 Meudon Principal Cedex, France

3 Center of Studies and Activities for Space, CISAS, G. Colombo, University of Padova, via Venezia 15, 35131 Padova, Italy

4 Research and Scientific Support Department, European Space Agency, 2201 Noordwijk, The Netherlands

5 Department of Physics \& Astronomy, Uppsala University, Box 516 75120 Uppsala, Sweden

6 Instituto de Astrofisica de Andalucia-CSIC, Glorieta de la Astronomia, 18008 Granada, Spain

7 German Aerospace Center (DLR), Institute of Planetary Research, Berlin-Adlershof, Germany

8 Physikalisches Institut, Sidlerstrasse 5, University of Bern, 3012 Bern, Switzerland

9 Department of Information Engineering, University of Padova, via Gradenigo 6/B, 35131 Padova, Italy

10 CNR-IFN UOS Padova LUXOR, via Trasea 7, 35131 Padova, Italy

11 Dipartimento di Fisica e Astronomia Galileo Galilei, University of Padova, vic. Osservatorio 3, 35122 Padova, Italy

12 Aix-Marseille Université, CNRS, LAM (Laboratoire d'Astrophysique de Marseille) UMR 7326, 13388 Marseille, France

13 Centro de Astrobiologia (INTA-CSIC), European Space Agency (ESA), European Space Astronomy Centre (ESAC), PO Box 78, 28691 Villanueva de la Canada, Madrid, Spain

14 International Space Science Institute, Hallerstrasse 6, 3012 Bern, Switzerland

15 PAS Space Research Center, Bartycka 18A, 00716 Warszawa, Poland

16 Department for Astronomy, University of Maryland, College Park, MD 20742-2421, USA

17 Gauss Professor Akademie der Wissenschaften zu Göttingen, 37077 Göttingen, Germany

18 LATMOS, CNRS/UVSQ/IPSL, 11 boulevard d'Alembert, 78280 Guyancourt, France

19 INAF Osservatorio Astronomico di Padova, Vicolo dell'Osservatorio 5, 35122 Padova, Italy

20 Department of Industrial Engineering University of Padova via Venezia, 1, 35131 Padova, Italy

21 University of Trento, via Sommarive, 9, 38123 Trento, Italy

22 INAF-Osservatorio Astronomico di Trieste, via Tiepolo 11, 34143 Trieste, Italy

23 Institute for Space Science, National Central University, 32054 Chung-Li, Taiwan

24 Institute for Geophysics and Extraterrestrial Physics, TU Braunschweig, 38106 Braunschweig, Germany

25 ESA/ESAC, PO Box 78, 28691 Villanueva de la Cañada, Spain

26 Observatory of the Hungarian Academy of Sciences, PO Box 67, 1525 Budapest, Hungary 


\section{Appendix A: USGS ISIS 3 with the OSIRIS data}

ISIS3 is an open source software, which supports many space missions, but Rosetta is not supported directly by USGS. Therefore, it was necessary to develop the supporting applications and data sets required by ISIS 3 to support OSIRIS data.

osiris2isis is an ISIS3 application that allows the conversion of OSIRIS PDS data into ISIS3 cub data format, ready for processing by ISIS3 applications. In addition to converting the binary data, osiris2isis also imports and translates the required PDS header entries to allow processing. Currently, this only supports full-frame images.

ISIS3 camera model libraries were also developed, another requirement of the ISIS3 application framework. Additionally, SPICE kernel databases were created, supporting data from the start of comet operations, and are continually updated to support new data as it is acquired by the spacecraft.

After the OSIRIS NAC images are imported to the ISIS3 using osiris 2 isis, further data processing steps use the standard commands of the software as described below:

1. Import of OSIRIS images in PDS format to ISIS3 software using osiris2isis.

2. Attaching SPICE information to each image using spiceinit.

3. Registration of the images using coreg.

4. Import of photometric angles extracted from the 3D shape to ISIS3 software using std2isis.

5. Photometric correction of registered images using photomet.

6. Generation of multispectral data using cubeit.

\section{Appendix B: Additional images}

\section{B.1. Activity seen from Hapi region}

Hapi region is located in between two lobes of the comet 67P. It was the most active region at the arrival to the comet (August 2014). An example of a large collimated jet feature is shown in Fig. B.1. More examples of the jets rising from Hapi region can be found in Lin et al. (2015).

\section{B.2. Activity detected from the pits}

The complete list of activity structures seen from the pits between August-October 2014 is compiled in Vincent et al. (2015) for the northern hemisphere of the comet. In Fig. B.2, examples of the activity rising from those pits are shown in Figs. $2 a$ and $b$ together with the activity rising from the clustered bright featured seen in Imhotep region (in Fig. 2c), seven months after the observations used in this study.

White square in Fig. 2c shows the location of the observation shown in Fig. 2c panel a. Blue squares indicate the location of

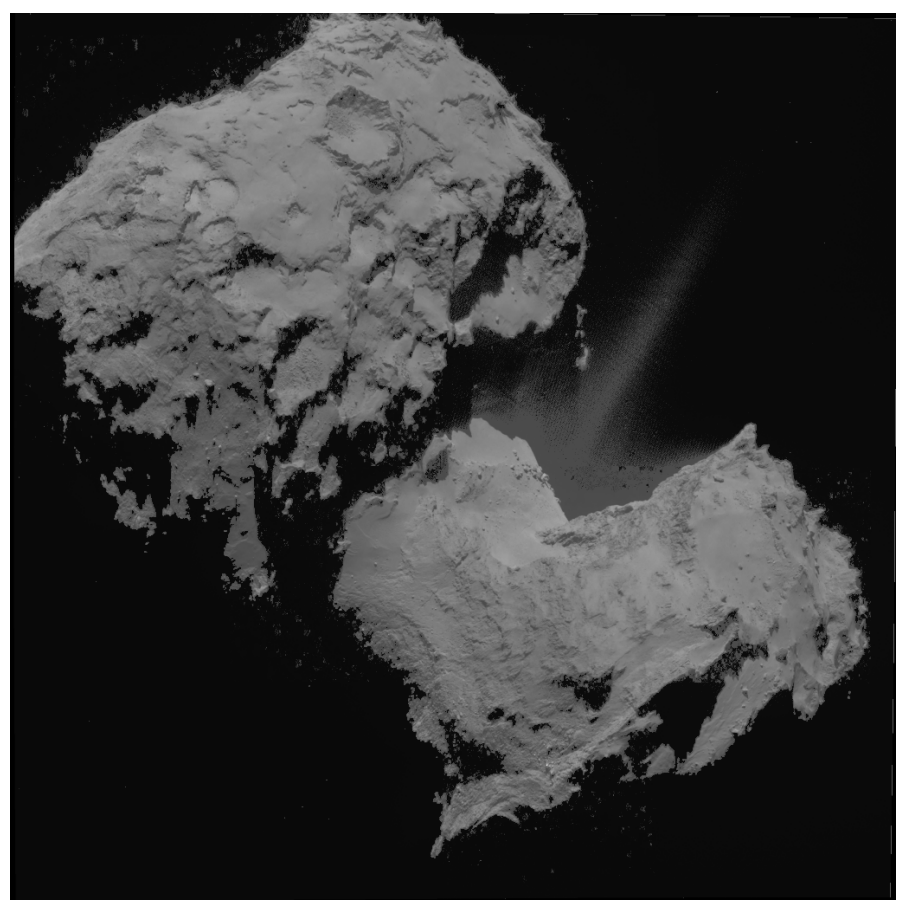

Fig. B.1. Activity seen in Hapi region on the image taken on 06 August 2014 at 04:19 UT. Stretched image is overlaid to show the large collimated jet.

the clustered bright features and the match between the observed jets. Figure 2c, panel d is the stretched version of Fig. $2 c$, panel c.

\section{B.3. Features on the images}

The geological features on the images are indicated in Fig. B.3 to support the main text in places where those features are mentioned. Activity is detected visually in Hapi, Seth, and Ma' at pits. Dust jet inversion of Vincent et al. (2016) also detects activity in Seth alcoves, the wall of Anuket alcove, in the region with roundish features in Imhotep, and in clustered bright features in Imhotep. Visual detection of jets rising from clustered bright features on Imhotep was carried out on 12 April 2015 images as seen in Fig. 2c. Additionally, stereoscopic reconstruction of the outburst observed on 12 March 2015 detected to be rising from the roundish feature terrain of Imhotep (Knollenberg et al. 2015).

\section{B.4. Clustered and isolated bright features on Imhotep}

Figure B.4 shows the bright features seen in the part of the Imhotep region covered by the images used in this study. 

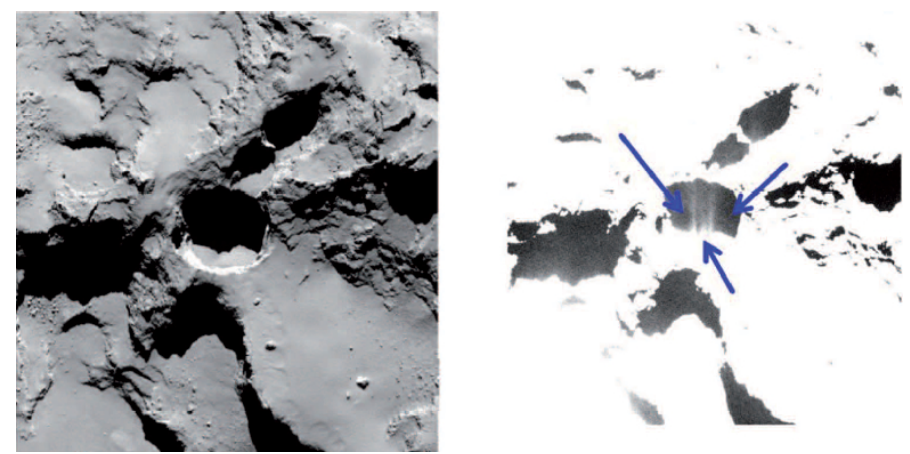

(a) Seth pits on the NAC image taken on 28 August 2014 at 20:42 UT (left panel) with the jets detected (right panel blue arrows in the stretched image).
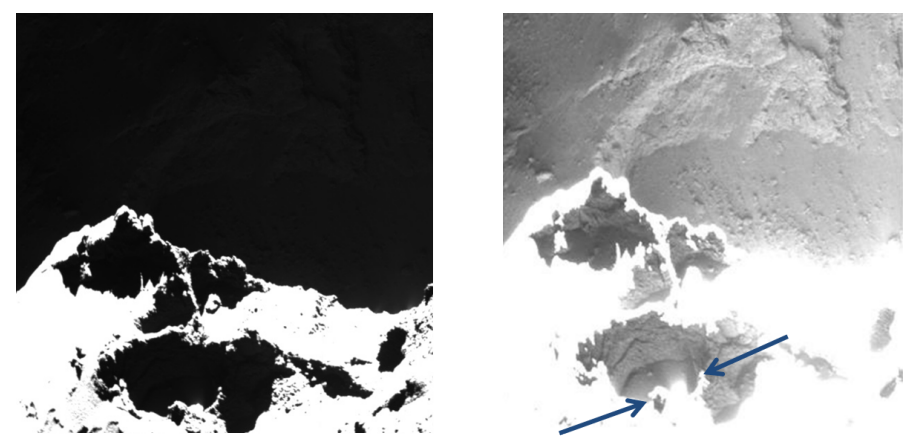

(b) Ma' at pit on the WAC image taken on 20 October 2014 at 04:52 UT (left panel) with a jet detected (right panel blue arrows in the stretched image).

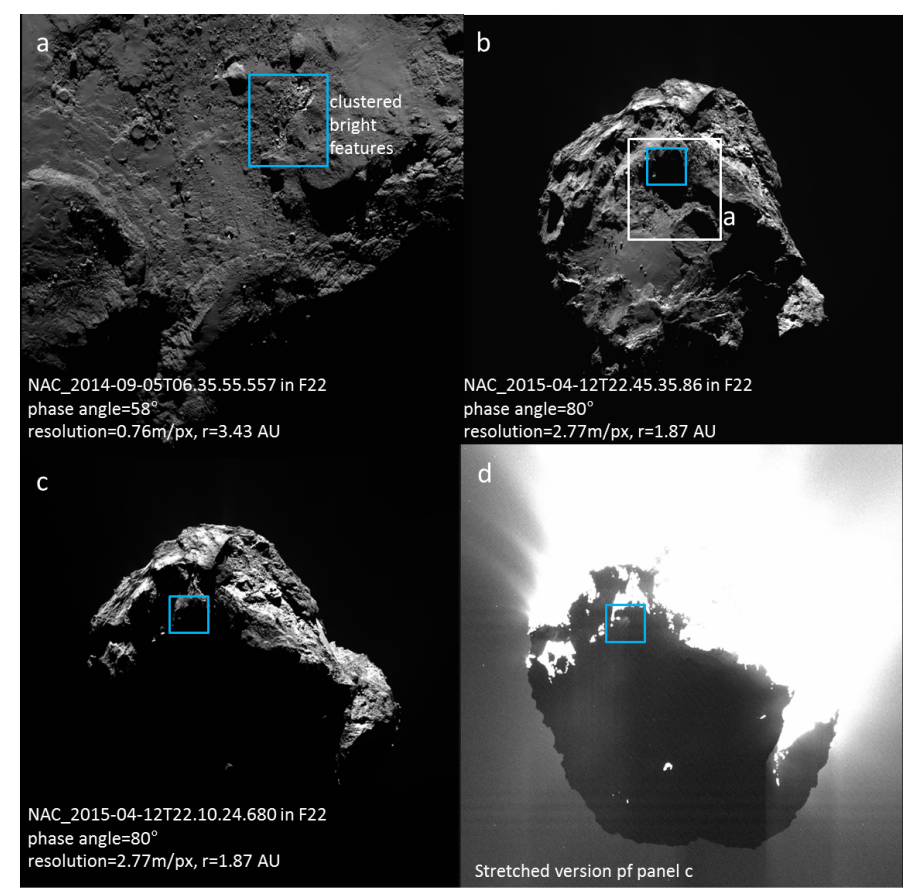

(c) Jets rising near clustered bright features of Imhotep.

Fig. B.2. Detected jets from the Seth pit (top panel), Ma'at pit (middle panel) and the clustered bright feature of Imhotep (bottom panel).

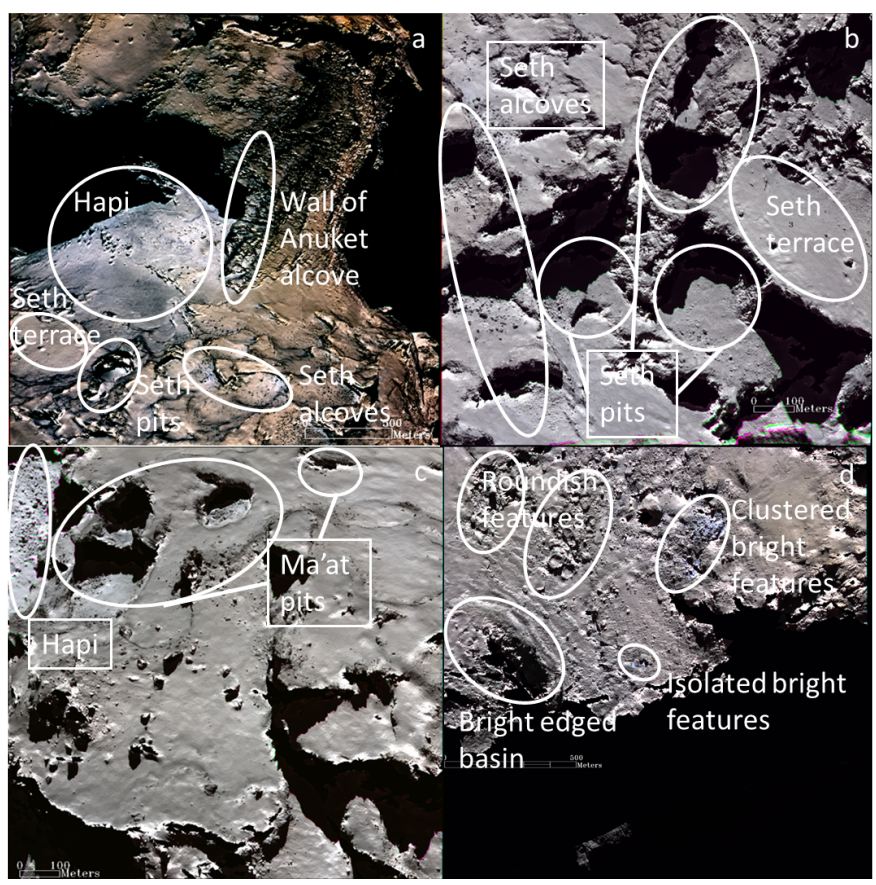

Fig. B.3. Geological features seen on the images.

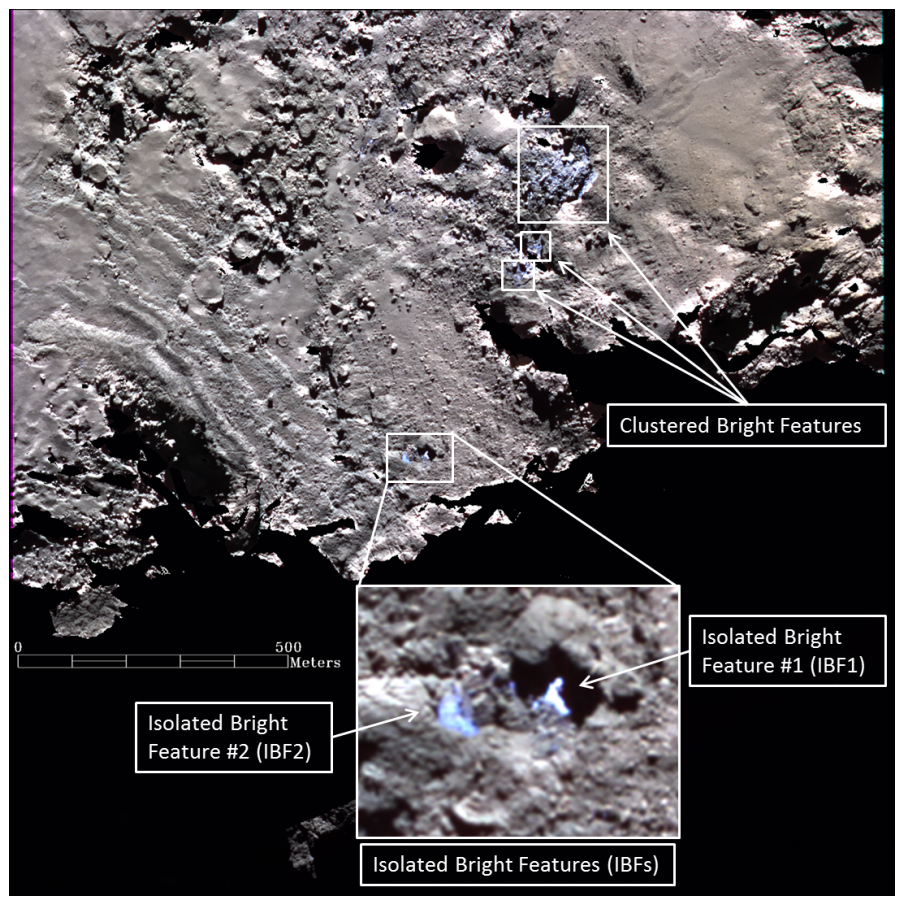

Fig. B.4. Clustered and isolated bright features of Imhotep. 


\section{Appendix C: Details of ROls}

The areas of the selected ROIs in Sect. 3 per image are presented in the Tables C.1-C.4.

Table C.1. ROIs defined in Fig. 3c for the Seth, Hapi, and Anuket regions.

\begin{tabular}{ccc}
\hline \hline ROI & $\begin{array}{c}\text { Number of } \\
\text { pixels }\end{array}$ & $\begin{array}{c}\text { Area } \\
\left(\mathrm{m}^{2}\right)\end{array}$ \\
\hline$\# 1$ & 5509 & 8066 \\
$\# 2$ & 152928 & 223902 \\
$\# 3$ & 71229 & 104286 \\
$\# 4$ & 406608 & 595315 \\
$\# 5$ & 148061 & 216776 \\
$\# 6$ & 360561 & 527897 \\
$\# 7$ & 410345 & 600768 \\
$\# 8$ & 86999 & 127373 \\
\hline
\end{tabular}

Table C.2. ROIs defined in Fig. 5c for the high resolution Seth pits.

\begin{tabular}{ccc}
\hline \hline ROI & $\begin{array}{c}\text { Number of } \\
\text { pixels }\end{array}$ & $\begin{array}{c}\text { Area } \\
\left(\mathrm{m}^{2}\right)\end{array}$ \\
\hline$\# 1$ & 12031 & 3253 \\
$\# 2$ & 148749 & 40222 \\
$\# 3$ & 333132 & 90079 \\
$\# 4$ & 348078 & 94120 \\
$\# 5$ & 401107 & 108459 \\
$\# 6$ & 58138 & 15720 \\
$\# 7$ & 78916 & 21339 \\
$\# 8$ & 196337 & 53089 \\
$\# 9$ & 39032 & 10554 \\
$\# 10$ & 88456 & 23918 \\
$\# 11$ & 63686 & 17220 \\
\hline
\end{tabular}

Table C.3. ROIs defined in Fig. 7c for the high resolution Ma'at region.

\begin{tabular}{ccc}
\hline \hline ROI & $\begin{array}{c}\text { Number of } \\
\text { pixels }\end{array}$ & $\begin{array}{c}\text { Area } \\
\left(\mathrm{m}^{2}\right)\end{array}$ \\
\hline$\# 1$ & 5670 & 1715 \\
$\# 2$ & 101001 & 30553 \\
$\# 3$ & 82982 & 25102 \\
$\# 4$ & 163072 & 49329 \\
$\# 5$ & 197295 & 59682 \\
$\# 6$ & 96736 & 29263 \\
$\# 7$ & 57650 & 17439 \\
$\# 8$ & 79113 & 23932 \\
$\# 9$ & 6226 & 1883 \\
$\# 10$ & 34415 & 10410 \\
\hline
\end{tabular}

Table C.4. ROIs defined in Fig. 9c for the Imhotep region.

\begin{tabular}{ccc}
\hline \hline ROI & $\begin{array}{c}\text { Number of } \\
\text { pixels }\end{array}$ & $\begin{array}{c}\text { Area } \\
\left(\mathrm{m}^{2}\right)\end{array}$ \\
\hline$\# 1$ & 8975 & 5184 \\
$\# 2$ & 726 & 419 \\
$\# 3$ & 2008 & 1160 \\
$\# 4$ & 52 & 30 \\
$\# 5$ & 102 & 59 \\
$\# 6$ & 4210 & 2432 \\
$\# 7$ & 5837 & 3371 \\
$\# 8$ & 4797 & 2771 \\
$\# 9$ & 10394 & 6004 \\
$\# 10$ & 12677 & 7322 \\
$\# 11$ & 28135 & 16251 \\
$\# 12$ & 8462 & 4888 \\
\hline
\end{tabular}

